\title{
STICKELBERGER ELEMENTS AND KOLYVAGIN SYSTEMS
}

\author{
KÂZIM BÜYÜKBODUK
}

\begin{abstract}
In this paper, we construct (many) Kolyvagin systems out of Stickelberger elements utilizing ideas borrowed from our previous work on Kolyvagin systems of Rubin-Stark elements. The applications of our approach are twofold. First, assuming Brumer's conjecture, we prove results on the odd parts of the ideal class groups of CM fields which are abelian over a totally real field, and we deduce Iwasawa's main conjecture for totally real fields (for totally odd characters). Although this portion of our results has already been established by Wiles unconditionally (and refined by Kurihara using an Euler system argument, when Wiles's work is assumed), the approach here fits well in the general framework the author has developed elsewhere to understand Euler/Kolyvagin system machinery when the core Selmer rank is $r>1$ (in the sense of Mazur and Rubin). As our second application, we establish a rather curious link between the Stickelberger elements and Rubin-Stark elements by using the main constructions of this article hand in hand with the "rigidity" of the collection of Kolyvagin systems proved by Mazur, Rubin, and the author.
\end{abstract}

\section{Contents}

1. Introduction . . . . . . . . . . . . . . . . . . . . . 123

2. Local conditions and Selmer groups . . . . . . . . . . . . . . . . . . . . . . 129

3. Euler systems from Stickelberger elements . . . . . . . . . . . . . . . . . . . . . . 149

4. Euler systems to Kolyvagin systems map . . . . . . . . . . . . . . . . . . . . 153

5. Applications .......................... . . 164

Acknowledgments . . . . . . . . . . . . . . . . . . . . . 172

References ........................... . . 172

\section{$\S 1$. Introduction}

The Euler/Kolyvagin system machinery is designed to bound the size of a Selmer group. In all well-known cases, the bounds obtained relate to

Received March 12, 2009. Revised November 30, 2009. Accepted February 11, 2011.

2010 Mathematics Subject Classification. Primary 11R23, 11R42; Secondary 11R27, 11R29, 11R34.

The author's work was partially supported by Marie Curie grant EU-FP7-IRG 230668 and Scientific and Technological Research Council of Turkey (TÜBİTAK) grant 109T662.

(C) 2011 by The Editorial Board of the Nagoya Mathematical Journal 
$L$-values and thus provide a link between arithmetic and analytic data. Wellknown prototypes for such a relation between arithmetic and analytic data are the Birch and Swinnerton-Dyer conjecture (more generally, Bloch-Kato conjectures) and the main conjectures of Iwasawa theory. The Kolyvagin system machinery has been successfully applied by many to obtain deep results towards proving these conjectures.

In [MR1], Howard, Mazur, and Rubin show that the existence of Kolyvagin systems relies on a cohomological invariant, what they call the core Selmer rank (see [MR1, Definition 4.1.11]). When the core Selmer rank is 1 , they determine the structure of the Selmer group completely in terms of a Kolyvagin system. However, when the core Selmer rank is greater than 1, not much could be said. One of the principal objectives of the current article is to explore this more mysterious case in depth for a particular Galois representation, for which the core Selmer rank is greater than 1.

Let $k$ be a totally real field of degree $[k: \mathbb{Q}]=r$, and let $G_{k}:=\operatorname{Gal}(\bar{k} / k)$ be its absolute Galois group. Fix once and for all an odd rational prime $p$, and let $\psi: G_{k} \rightarrow \mathbb{Z}_{p}^{\times}$be a totally even character which has finite prime-to- $p$ order. Consider the $G_{k}$-representation $T^{\prime}:=\mathbb{Z}_{p}(1) \otimes \psi^{-1}$. The core Selmer rank of the Galois representation $T^{\prime}$ is $r$, and $T^{\prime}$ leads us to one of the basic instances when the core Selmer rank is greater than 1. In [B1] and [B2], the author studied the Kolyvagin system machinery for $T^{\prime}$. The main idea in these two papers is to modify the relevant Selmer group appropriately and construct Kolyvagin systems out of Rubin-Stark elements defined in [R2] so as to control this modified Selmer group. In this paper, we consider the $G_{k}$-representation $T=\mathbb{Z}_{p}(\chi)$, where $\chi: G_{k} \rightarrow \mathbb{Z}_{p}^{\times}$is a totally odd character which has finite prime-to- $p$ order. The $G_{k}$-representation $T$ turns out to have core Selmer rank $r$ as well. As in [B1] and [B2], we introduce certain modified Selmer groups associated with the representation $T$. In this setting, the Euler system that gives rise to the Kolyvagin system which controls the modified Selmer group is obtained from the Stickelberger elements.

Before we state the main results of this article, we set some notation which will be in effect throughout the paper. Let $p, k, G_{k}$, and $r$ be as above, and let $\chi: G_{k} \rightarrow \mathbb{Z}_{p}^{\times}$be a totally odd character, which is different from the Teichmüller character $\omega$ that gives the action of $G_{k}$ on the $p$ th roots of unity. Let $k_{\infty}$ denote the cyclotomic $\mathbb{Z}_{p}$-extension of $k$. Consider the following properties.

(A1) For any prime $\wp$ of $k$ above $p$, we have $\chi(\wp) \neq 1$. 
(A2) Any prime of $k$ above $p$ totally ramifies in $k_{\infty} / k$.

Note that (A2) is true, for example, if $k / \mathbb{Q}$ is unramified. Hypothesis (A1) ensures that the associated Deligne-Ribet $p$-adic $L$-function $\mathcal{L}_{\omega \chi^{-1}}$ does not have a trivial zero in the sense of [G]. Note that Wiles in [W2] gave a proof of the main conjecture without assuming these two hypotheses.

Let $L$ be the fixed field of $\operatorname{ker}(\chi)$ inside a fixed algebraic closure $\bar{k}$ of $k$; write $\Delta=\operatorname{Gal}(L / k)$. For any number field $K$ containing $L$, let $A_{K}$ be the $p$-part of the ideal class group of $K$, and let $A_{K}^{\chi}$ be its $\chi$-isotypic part. We fix $S$ as the set of places of $k$ which consists of all infinite places of $k$, all places $\lambda$ which divide the conductor $\mathfrak{f}_{\chi}$ of $\chi$, and all the places of $k$ above $p$. Finally, let $\theta_{L, S}=\theta_{L} \in \mathbb{Z}_{p}[\Delta]$ be the Stickelberger element (defined precisely in $[\mathrm{Ku}$, Section 1.2]; see also Section 3 below) relative to $S$. For the main results of this article, we will assume the $\chi$-part of Brumer's conjecture, as follows.

Assumption 1.1. $\theta_{K}^{\chi}$ annihilates $A_{K}^{\chi}$.

We remark here that Wiles [W1] proved that Brumer's conjecture as stated above follows from his proof in [W2] of the main conjecture of Iwasawa theory for totally real fields. In this paper, we prove the other way around, namely, that, assuming Brumer's conjecture, one might also prove the main conjecture (see Theorem B below; see [Ku], where Kurihara refines Wiles's result using a different type of Euler system argument).

The first application of the treatment here is Theorem 5.3 below.

Theorem A. Suppose that hypothesis (A1) and Assumption 1.1 hold. Then,

$$
\left|A_{L}^{\chi}\right|=\left|\mathbb{Z}_{p} / \chi\left(\theta_{L}\right) \mathbb{Z}_{p}\right|
$$

With a bit more work, we can prove the Iwasawa theoretic version of Theorem A, which we state below. Set $\Gamma=\operatorname{Gal}\left(k_{\infty} / k\right)$, and set $\Lambda=\mathbb{Z}_{p}[[\Gamma]]$, as usual. Let $\mathcal{L}_{\omega \chi^{-1}} \in \Lambda$ denote the Deligne-Ribet $p$-adic $L$-function (see $[\mathrm{DR}])$. We recall in (5.2) the basic interpolation property which characterizes $\mathcal{L}_{\omega \chi^{-1}}$. Let $\mathrm{Tw}_{\left\langle\rho_{\text {cyc }}\right\rangle}$ be a certain twisting operator on $\Lambda$ (see Section 5.2 below for its definition). For any abelian group $A$, let $A^{\vee}:=\operatorname{Hom}\left(A, \mathbb{Q}_{p} / \mathbb{Z}_{p}\right)$ denote its Pontryagin dual, and finally, let $\operatorname{char}(M)$ denote the characteristic ideal of a finitely generated $\Lambda$-module $M$ (with the convention that $\operatorname{char}(M)=0$ unless $M$ is $\Lambda$-torsion). Then we are able to prove the following (see Theorem 5.8 and Corollary 5.10 for a slightly improved version so as to include the case $\left.\boldsymbol{\mu}_{p} \subset L\right)$. 
Theorem B. Suppose that hypotheses (A1) and (A2) as well as Assumption 1.1 hold. Assume also that $L$ does not contain pth roots of unity. Then,

$$
\operatorname{char}\left(\left(\underset{n}{\left(\lim _{\longrightarrow}\right.} A_{L_{n}}^{\chi}\right)^{\vee}\right)=\operatorname{Tw}_{\left\langle\rho_{\text {cyc }}\right\rangle}\left(\mathcal{L}_{\omega \chi^{-1}}\right) .
$$

These results have already been obtained by Wiles [W2] without appealing to the Euler system machinery, even without the assumptions of Theorem B above. Kurihara $[\mathrm{Ku}]$ proved a refined version of Theorem B using an Euler system argument (still building on Wiles's results), though significantly different than ours. The novelty in this paper is a new treatment of the Euler/Kolyvagin system machinery via what we call $\mathcal{L}$-restricted Euler systems (see Section 4.1, especially Remark/Definition 4.10 and Example 4.11), when the core Selmer rank (in the sense of Mazur and Rubin [MR1]) of the Galois representation in question is $r>1$. One benefit of the new approach presented in this paper is a rather surprising link between Stickelberger elements and the (conjectural) Rubin-Stark elements, which we prove below in Theorem 5.16; see also Theorem $\mathrm{C}$ in this section and the paragraph that follows its statement.

As in our earlier papers ([B1], [B2]), we improve the Euler/Kolyvagin system machinery of [R3], [P2], [Ka], and [MR1] to prove Theorems A and $\mathrm{B}$, generalizing Rubin's treatment in [R3, Section 3.4] of the Stickelberger element Euler system. The main obstruction to apply the machinery of [MR1] directly in our setting is the fact that when $r>1$, what Mazur and Rubin call the canonical Selmer structure in [MR1] produces a Selmer group too big to control using the theory developed there. In order to deal with this matter, we proceed as follows.

(1) We first "refine" the canonical Selmer structure by introducing more restrictive local conditions at $p$. We achieve this by choosing a line $\mathcal{L}$ inside a certain local cohomology group at $p$ (see Section 2.3). This step has to do with the issue discussed in Remark 3.5(i) below.

(2) We introduce what we call the module of $\mathcal{L}$-restricted Euler systems (see Definition 4.10). The point in doing so is that an Euler system (in the sense of Rubin) a priori gives rise to a Kolyvagin system only for the canonical Selmer structure, and not necessarily for the refined Selmer structure defined in Step (1). On the other hand, as we verify here, an $\mathcal{L}$-restricted Euler system does give rise to a Kolyvagin system for the refined Selmer structure. 
(3) We then obtain $\mathcal{L}$-restricted Euler systems starting from Stickelberger elements (see Example 4.11), which we apply to deduce Theorems A and $\mathrm{B}$ above. For this part, one needs to determine the structure of semilocal cohomology groups and, using this information, choose a useful collection of homomorphisms (see Proposition 4.9). The choice of such a collection also appears in [R3, Proposition D.1.3], where Rubin explicitly constructs one, though in a different way from ours; see Remark 3.5 for a comparison of our construction here to Rubin's work in the case $k=\mathbb{Q}[$ R3, Section III.4]. Rubin's construction is useful only when the base field $k$ is $\mathbb{Q}$, whereas we abstractly show that a collection of homomorphisms with the necessary properties exists for an arbitrary totally real base field $k$.

The Galois representation (and the Euler system attached to it) which we treat in this paper needs to be handled in a slightly different manner than the case of Rubin-Stark elements (which was studied in [B1], [B2]), as far as the Euler/Kolyvagin system machinery is concerned. In a forthcoming paper [B4], the setup from the current article and that from [B3] are combined to treat the theory of Kolyvagin systems which descend from Euler systems* for an arbitrary self-dual Galois representation whose core Selmer rank is $r>1$.

Our method to improve the results of [P2], [R3], and [MR1] relies on the choice of a rank 1 direct summand (which we call $\mathcal{L}$ above) inside the semilocal cohomology group at $p$. This makes our approach seem less natural. We address this issue in Remark 2.32 and show that the module generated by the "leading terms" of the Kolyvagin systems constructed this way does not depend on the decomposition of the semilocal cohomology group at $p$ into rank 1 direct summands (see Theorem 2.33).

Besides the standard applications (i.e., Theorems A and B above) of our construction of what we call an $\mathcal{L}$-restricted Kolyvagin system (see Definition 2.36, Theorem 4.15, and Remark 4.8) out of Stickelberger elements, we also prove the following statement regarding the local Iwasawa theory of Rubin-Stark elements, which was proved in [B2] assuming the truth of the main conjecture.

TheOREM C. Let $\psi: G_{k} \rightarrow \mathbb{Z}_{p}^{\times}$be a totally even character. Suppose that both $\psi$ and $\chi=\omega \psi^{-1}$ satisfy hypothesis (A1), and assume that (A2) holds

${ }^{*}$ More precisely, Euler systems of rank $r$ in the terminology of [P2]. 
as well as Assumption 1.1. Then,

$$
\operatorname{char}\left(\wedge_{\Lambda}^{r} H^{1}\left(k_{p}, T^{\prime} \otimes \Lambda\right) / \Lambda \cdot c_{k_{\infty}}^{\psi}\right)=\mathcal{L}_{\psi}
$$

Here, $T^{\prime}=\mathbb{Z}_{p}(1) \otimes \psi^{-1}$, and $c_{k_{\infty}}^{\psi}:=\left\{c_{k_{n}}^{\psi}\right\}$ is the $\psi$-part of the collection of (conjectural) Rubin-Stark elements (which we assume to exist) along the cyclotomic tower (see [B2, Section 3] for a precise definition of these elements).

In fact, we are able to prove considerably more than Theorem $\mathrm{C}$ in regard of the Rubin-Stark elements. In Theorem 5.16(i), we obtain a relation between the Stickelberger elements and Rubin-Stark elements (note that the existence of the latter is conjectural), making use of the formalism of $\mathcal{L}$-restricted Euler systems we develop in this paper, as well as the rigidity of the module of $\Lambda$-adic Kolyvagin systems proved in [B3]. This, we believe, is interesting on its own right. We also note that the "rigidity phenomenon" which plays an important role for the link we obtain here (between the Stickelberger elements and Rubin-Stark elements) has recently been utilized by Mazur and Rubin [MR2] (in a rather similar fashion) to prove an important portion of Darmon's refinement of Gross's conjecture.

We finally remark that, thanks to (an appropriate variant of) Proposition 4.5, we may bypass the need to appeal to Krasner's lemma in [B1] and [B2], and hence we may remove the hypothesis that $\chi$ is unramified at all primes $\wp \subset k$ above $p$ on the main results of [B1] and [B2].

Notation. Besides what we have fixed above, the following notation will be in effect throughout.

For any field $F$, let $G_{F}$ denote the Galois group of a fixed separable closure $\bar{F}$ of $F$. For any abelian group $A$, write

$$
A^{\wedge}:=\operatorname{Hom}\left(\operatorname{Hom}\left(A, \mathbb{Q}_{p} / \mathbb{Z}_{p}\right), \mathbb{Q}_{p} / \mathbb{Z}_{p}\right)
$$

for its $p$-adic completion. Suppose in addition that $\Delta$ acts on $A$; we then write $A^{\chi}$ for the $\chi$-isotypic component of $A^{\wedge}$.

For $k_{\infty} / k$ as above, let $k_{n} / k$ be the unique subfield of degree $p^{n}$. We set $\Gamma_{n}=\operatorname{Gal}\left(k_{n} / k\right)$, and we write $L_{n}=L k_{n}$. For any prime $\mathfrak{q} \subset k$, let $k(\mathfrak{q})$ denote the $p$-part of the ray class field extension of $k$ modulo $\mathfrak{q}$. For any square-free integral ideal $\mathfrak{q}_{1} \cdots \mathfrak{q}_{n}=\tau \subset k$, we set $k(\tau)$ as the composite

$$
k(\tau)=k\left(\mathfrak{q}_{1}\right) \cdots k\left(\mathfrak{q}_{n}\right) .
$$

Set $\Delta_{\tau}=\operatorname{Gal}(k(\tau) / k)$. We let $L(\tau)=L k(\tau), k_{n}(\tau)=k_{n} k(\tau)$, and $L_{n}(\tau)=$ $k_{n} L(\tau)$. 


\section{§2. Local conditions and Selmer groups}

Much of this section is a review of the Kolyvagin system machinery and the terminology of [MR1], which we will employ for the remainder of this paper. The reader who is comfortable with the language of [MR1] may safely jump to Section 3 after a glance at Sections 2.3.1 and 2.3.2 and then at Proposition 2.21, Corollary 2.22, Propositions 2.23 and 2.29, and Theorem 2.37. We also note that Remark 2.32 (esp. Theorem 2.33) should be of interest for the general understanding of the Kolyvagin system machinery when the core Selmer rank is greater than 1.

\subsection{Selmer structures on $T=\mathbb{Z}_{p}(\chi)$}

Below we use the notation that was set in Section 1 . Recall that $\Gamma:=$ $\operatorname{Gal}\left(k_{\infty} / k\right)$ and $\Lambda:=\mathbb{Z}_{p}[[\Gamma]]$ is the cyclotomic Iwasawa algebra.

We first recall Mazur and Rubin's definition in [MR1, Definition 2.1.1] of a Selmer structure, in particular, the canonical Selmer structure on $T$ and $T \otimes \Lambda$.

2.1.1. Local conditions. Let $R$ be a complete local Noetherian ring, and let $M$ be an $R\left[\left[G_{k}\right]\right]$-module which is free of finite rank over $R$. In this paper, we are interested in the case when $R=\Lambda$ or its certain quotients, and when $M$ is $T \otimes \Lambda$ or its corresponding quotients by ideals of $\Lambda$. For example, if we start with the Galois representation $T \otimes \Lambda$ with coefficients in $\Lambda$, we get the representation $T$ upon taking the quotient of $T \otimes \Lambda$ by the augmentation ideal of $\Lambda$.

For each place $\lambda$ of $k$, a local condition $\mathcal{F}$ (at $\lambda$ ) on $M$ is a choice of an $R$-submodule $H_{\mathcal{F}}^{1}\left(k_{\lambda}, M\right)$ of $H^{1}\left(k_{\lambda}, M\right)$. A local condition $\mathcal{F}$ at $p$ is a choice of an $R$-submodule $H_{\mathcal{F}}^{1}\left(k_{p}, M\right)$ of the semilocal cohomology group $H^{1}\left(k_{p}, M\right):=\bigoplus_{\wp \mid p} H^{1}\left(k_{\wp}, M\right)$, where the direct sum is over all the primes $\wp$ of $k$ which lie above $p$.

For examples of local conditions, see [MR1, Definitions 1.1.6 and 3.2.1].

Suppose that $\mathcal{F}$ is a local condition (at $\lambda$ ) on $M$. If $M^{\prime}$ is a submodule of $M$ (resp., $M^{\prime \prime}$ is a quotient module), then $\mathcal{F}$ induces local conditions (which we still denote by $\mathcal{F}$ ) on $M^{\prime}$ (resp., on $M^{\prime \prime}$ ) by taking $H_{\mathcal{F}}^{1}\left(k_{\lambda}, M^{\prime}\right.$ ) (resp., $\left.H_{\mathcal{F}}^{1}\left(k_{\lambda}, M^{\prime \prime}\right)\right)$ to be the inverse image (resp., the image) of $H_{\mathcal{F}}^{1}\left(k_{\lambda}, M\right)$ under the natural maps induced by

$$
M^{\prime} \hookrightarrow M, \quad M \rightarrow M^{\prime \prime} .
$$


Definition 2.1. Propagation of a local condition $\mathcal{F}$ on $M$ to a submodule $M^{\prime}$ (and a quotient $M^{\prime \prime}$ of $M$ ) is the local condition $\mathcal{F}$ on $M^{\prime}$ (and on $M^{\prime \prime}$ ) obtained following the procedure above.

For example, if $I$ is an ideal of $R$, then a local condition on $M$ induces local conditions on $M / I M$ and $M[I]$, by propagation.

2.1.2. Selmer structures and Selmer groups. Notation from Section 2.1.1 is in effect throughout this section. We will denote $G_{k_{\lambda}}$ by $\mathcal{D}_{\lambda}$ whenever we wish to identify this group with a closed subgroup of $G_{k}$, namely, with a particular decomposition group at $\lambda$. We further define $\mathcal{I}_{\lambda} \subset \mathcal{D}_{\lambda}$ to be the inertia group and $\operatorname{Fr}_{\lambda} \in \mathcal{D}_{\lambda} / \mathcal{I}_{\lambda}$ to be the arithmetic Frobenius element at $\lambda$.

Definition 2.2. A Selmer structure $\mathcal{F}$ on $M$ is a collection of the following data:

- a finite set $\Sigma(\mathcal{F})$ of places of $k$, including all infinite places and primes above $p$, and all primes where $M$ is ramified,

- for every $\lambda \in \Sigma(\mathcal{F})$, a local condition (in the sense of Section 2.1.1) on $M$ (which we view now as an $R\left[\left[\mathcal{D}_{\lambda}\right]\right]$-module), that is, a choice of $R$ submodule

$$
H_{\mathcal{F}}^{1}\left(k_{\lambda}, M\right) \subset H^{1}\left(k_{\lambda}, M\right) .
$$

If $\lambda \notin \Sigma(\mathcal{F})$, we will also write $H_{\mathcal{F}}^{1}\left(k_{\lambda}, M\right)=H_{\mathrm{f}}^{1}\left(k_{\lambda}, M\right)$, where the module $H_{\mathrm{f}}^{1}\left(k_{\lambda}, M\right)$ is the finite part of $H^{1}\left(k_{\lambda}, M\right)$, defined as in [MR1, Definition 1.1.6].

Definition 2.3. Define the Cartier dual of $M$ to be the $R\left[\left[G_{k}\right]\right]$-module

$$
M^{*}:=\operatorname{Hom}\left(M, \mu_{p} \infty\right),
$$

where $\mu_{p} \infty$ stands for the $p$-power roots of unity.

Let $\lambda$ be a prime of $k$. There is a perfect pairing

$$
<,>_{\lambda}: H^{1}\left(k_{\lambda}, M\right) \times H^{1}\left(k_{\lambda}, M^{*}\right) \longrightarrow H^{2}\left(k_{\lambda}, \mu_{p^{\infty}}\right) \stackrel{\sim}{\longrightarrow} \mathbb{Q}_{p} / \mathbb{Z}_{p}
$$

called the local Tate pairing.

Definition 2.4. The dual local condition $\mathcal{F}^{*}$ on $M^{*}$ of a local condition $\mathcal{F}$ on $M$ is defined so that $H_{\mathcal{F}^{*}}^{1}\left(k_{\lambda}, M^{*}\right)$ is the orthogonal complement of $H_{\mathcal{F}}^{1}\left(k_{\lambda}, M\right)$ with respect to the local Tate pairing $<,>_{\lambda}$.

The dual Selmer structure $\mathcal{F}^{*}$ is defined by setting $\Sigma\left(\mathcal{F}^{*}\right)=\Sigma(\mathcal{F})$ and choosing the local conditions on $M^{*}$ as the dual local conditions $H_{\mathcal{F}^{*}}^{1}\left(k_{\lambda}, M^{*}\right)=H_{\mathcal{F}}^{1}\left(k_{\lambda}, M\right)^{\perp}$ at every prime $\lambda \in \Sigma\left(\mathcal{F}^{*}\right)$. 
Definition 2.5. If $\mathcal{F}$ is a Selmer structure on $M$, we define the Selmer module $H_{\mathcal{F}}^{1}(k, M)$ to be the kernel of the sum of the restriction maps

$$
H_{\mathcal{F}}^{1}(k, M):=\operatorname{ker}\left(H^{1}\left(\operatorname{Gal}\left(k_{\Sigma(\mathcal{F})} / k\right), M\right) \rightarrow \bigoplus_{\lambda \in \Sigma(\mathcal{F})} \frac{H^{1}\left(k_{\lambda}, M\right)}{H_{\mathcal{F}}^{1}\left(k_{\lambda}, M\right)}\right)
$$

Here, $k_{\Sigma(\mathcal{F})}$ is the maximal extension of $k$ which is unramified outside $\Sigma(\mathcal{F})$. We also define the dual Selmer module $H_{\mathcal{F}^{*}}^{1}\left(k, M^{*}\right)$ in a similar fashion: just replace $M$ by $M^{*}$ and $\mathcal{F}$ by $\mathcal{F}^{*}$ in $(2.1)$.

ExAmple 2.6. In this example, we recall [MR1, Definitions 3.2.1, 5.3.2].

(i) Let $R=\mathbb{Z}_{p}$, and let $M$ be a free $R$-module endowed with a continuous action of $G_{k}$, which is unramified outside a finite set of places of $k$. We define a Selmer structure $\mathcal{F}_{\text {can }}$ on $M$ by setting

$-\Sigma\left(\mathcal{F}_{\text {can }}\right)=\{\lambda: M$ is ramified at $\lambda\} \cup\{\wp \mid p\} \cup\{v \mid \infty\}$,

$-H_{\mathcal{F}_{\text {can }}}^{1}\left(k_{\lambda}, M\right)= \begin{cases}H_{\mathrm{f}}^{1}\left(k_{\lambda}, M\right) & \text { if } \lambda \in \Sigma\left(\mathcal{F}_{\text {can }}\right), \lambda \nmid p \infty, \\ H^{1}\left(k_{\lambda}, M\right) & \text { if } \lambda \mid p .\end{cases}$

Here, $H_{\mathrm{f}}^{1}\left(k_{\lambda}, M\right):=\operatorname{ker}\left\{H^{1}\left(k_{\lambda}, M\right) \rightarrow H^{1}\left(k_{\lambda}, M \otimes \mathbb{Q}_{p}\right)\right\}$ for every $\lambda \nmid$ $p \mathfrak{f}_{\chi}$.

The Selmer structure $\mathcal{F}_{\text {can }}$ is called the canonical Selmer structure on $M$.

(ii) Now let $R=\Lambda$ be the cyclotomic Iwasawa algebra, and let $\mathbb{M}$ be a free $R$-module endowed with a continuous action of $G_{k}$, which is unramified outside a finite set of places of $k$. We define a Selmer structure $\mathcal{F}_{\Lambda}$ on $\mathbb{M}$ by setting

$-\Sigma\left(\mathcal{F}_{\Lambda}\right)=\{\lambda: \mathbb{M}$ is ramified at $\lambda\} \cup\{\wp \mid p\} \cup\{v \mid \infty\}$,

- $H_{\mathcal{F}_{\Lambda}}^{1}\left(k_{\lambda}, \mathbb{M}\right)=H^{1}\left(k_{\lambda}, \mathbb{M}\right)$ for $\lambda \in \Sigma\left(\mathcal{F}_{\Lambda}\right)$.

The Selmer structure $\mathcal{F}_{\Lambda}$ is called the canonical Selmer structure on $\mathbb{M}$.

As in Definition 2.1, the induced Selmer structure on the quotients $\mathbb{M} / I \mathbb{M}$ is still denoted by $\mathcal{F}_{\Lambda}$. Note that $H_{\mathcal{F}_{\Lambda}}^{1}\left(k_{\lambda}, \mathbb{M} / I \mathbb{M}\right)$ will not usually be the same as $H^{1}\left(k_{\lambda}, \mathbb{M} / I \mathbb{M}\right)$. In particular, when $I$ is the augmentation ideal inside $\Lambda, \mathcal{F}_{\Lambda}$ on $\mathbb{M}$ will not always propagate to $\mathcal{F}_{\text {can }}$ on $M=\mathbb{M} \otimes \Lambda / I$.

However, when $M=T$ and $\mathbb{M}=T \otimes \Lambda$ as in Section 1, it is not hard to see that $\mathcal{F}_{\Lambda}$ does propagate to $\mathcal{F}_{\text {can }}$.

REMARK 2.7. When $R=\Lambda$ and $\mathbb{M}=T \otimes \Lambda$ with $T=\mathbb{Z}_{p}(\chi)$, we will see in Section 2.5.2 that the Selmer structure $\mathcal{F}_{\text {can }}$ of [B3, Section 2.1] on the quotients $T \otimes \Lambda /(f)$ may be identified, under hypothesis (A1) on $\chi$, with 
the propagation of $\mathcal{F}_{\Lambda}$ to the quotients $T \otimes \Lambda /(f)$, for every distinguished polynomial $f$ inside $\Lambda$.

Definition 2.8. A Selmer triple is a triple $(M, \mathcal{F}, \mathcal{P})$ where $\mathcal{F}$ is a Selmer structure on $M$ and $\mathcal{P}$ is a set of rational primes, disjoint from $\Sigma(\mathcal{F})$.

REMARK 2.9. In our setting, that is, when the Galois representation in question is $T \otimes \Lambda$ or its quotients by ideals of $\Lambda$, one may explicitly compute the cohomology groups in terms of certain groups of homomorphisms (see [R3, Sections I.6.1-3]). Nevertheless, we will insist on using the cohomological language for the sake of notational consistency with [MR1] from which we borrow the main technical results. We also hope that the similarity of the ideas applied here and in [B1] and [B2] is more apparent this way.

\subsection{Computing Selmer groups explicitly}

In this section, we give an explicit description of the Selmer groups for the $G_{k}$-representations $T=\mathbb{Z}_{p}(\chi)$ (resp., for $T \otimes \Lambda$ ) and for $T^{*}=\boldsymbol{\mu}_{p^{\infty}} \otimes \chi^{-1}$ (resp., for $\left.(T \otimes \Lambda)^{*}\right)$, following [R3, Section I.6.2] and [MR1, Section 6.1].

2.2.1. Selmer groups over $k$. Recall that $L$ is the CM field cut by $\chi$. For any $m \in \mathbb{Z}^{+}$, it follows (as in [MR1, Section 6.1]) from the inflation-restriction sequence that

$$
H^{1}\left(k, T / p^{m} T\right)=H^{1}\left(k, \mathbb{Z} / p^{m} \mathbb{Z}(\chi)\right) \cong \operatorname{Hom}\left(G_{L}, \mathbb{Z} / p^{m} \mathbb{Z}\right)^{\chi^{-1}},
$$

and similarly for every prime $\lambda$ of $k$,

$$
H^{1}\left(k_{\lambda}, T / p^{m} T\right) \cong\left(\bigoplus_{\mathfrak{q} \mid \lambda} \operatorname{Hom}\left(G_{L_{\mathfrak{q}}}, \mathbb{Z} / p^{m} \mathbb{Z}\right)\right)^{\chi^{-1}} .
$$

Therefore, for the semilocal cohomology at a rational prime $\ell$, we have

$$
H^{1}\left(k_{\ell}, T / p^{m} T\right) \cong \bigoplus_{\lambda \mid \ell}\left(\bigoplus_{\mathfrak{q} \mid \lambda} \operatorname{Hom}\left(G_{L_{\mathfrak{q}}}, \mathbb{Z} / p^{m} \mathbb{Z}\right)\right)^{\chi^{-1}}
$$

Passing to the inverse limit, we obtain

$$
H^{1}(k, T) \cong \operatorname{Hom}\left(G_{L}, \mathbb{Z}_{p}\right)^{\chi^{-1}}
$$

and

$$
H^{1}\left(k_{\ell}, T\right) \cong \bigoplus_{\lambda \mid \ell}\left(\bigoplus_{\mathfrak{q} \mid \lambda} \operatorname{Hom}\left(G_{L_{\mathfrak{q}}}, \mathbb{Z}_{p}\right)\right)^{\chi^{-1}}
$$


For the dual representation $T^{*}$, we have by the inflation-restriction sequence and by Kummer theory

$$
H^{1}\left(k, T^{*}\left[p^{m}\right]\right)=H^{1}\left(k, \boldsymbol{\mu}_{p^{m}} \otimes \chi^{-1}\right) \cong\left(L^{\times} /\left(L^{\times}\right)^{p^{m}}\right)^{\chi},
$$

and similarly for every prime $\lambda \subset k$,

$$
H^{1}\left(k_{\lambda}, T^{*}\left[p^{m}\right]\right) \cong\left(L_{\lambda}^{\times} /\left(L_{\lambda}^{\times}\right)^{p^{m}}\right)^{\chi} .
$$

Also, for the semilocal cohomology, we have

$$
H^{1}\left(k_{\ell}, T^{*}\left[p^{m}\right]\right) \cong\left(L_{\ell}^{\times} /\left(L_{\ell}^{\times}\right)^{p^{m}}\right)^{\chi},
$$

where $L_{\lambda}:=L \otimes_{k} k_{\lambda}$, the sum of the completions of $L$ at the primes above $\lambda$, and $L_{\ell}:=L \otimes_{\mathbb{Q}} \mathbb{Q}_{\ell}$. Taking direct limits, we see that

$$
H^{1}\left(k, T^{*}\right) \cong\left(L^{\times} \otimes \mathbb{Q}_{p} / \mathbb{Z}_{p}\right)^{\chi}
$$

and

$$
H^{1}\left(k_{\ell}, T^{*}\right) \cong\left(L_{\ell}^{\times} \otimes \mathbb{Q}_{p} / \mathbb{Z}_{p}\right)^{\chi}
$$

Proposition 2.10. The canonical Selmer structure $\mathcal{F}_{\text {can }}$ on $T$ (resp., $\mathcal{F}_{\text {can }}^{*}$ on $\left.T^{*}\right)$ is given by

- $\Sigma\left(\mathcal{F}_{\text {can }}\right)=\Sigma\left(\mathcal{F}_{\text {can }}^{*}\right)=\left\{\lambda: \lambda \mid p \mathfrak{f}_{\chi}\right\} \cup\{v \mid \infty\}$

and by setting (using the identifications above)

- $H_{\mathcal{F}_{\text {can }}}^{1}\left(k_{\ell}, T\right)=\left(\bigoplus_{\mathfrak{q} \mid \ell} \operatorname{Hom}\left(G_{L_{\mathfrak{q}}} / \mathcal{I}_{\mathfrak{q}}, \mathbb{Z}_{p}\right)\right)^{\chi^{-1}}$,

$H_{\mathcal{F}_{\text {can }}^{*}}^{1}\left(k_{\ell}, T^{*}\right)=\left(\mathcal{O}_{L, \ell}^{\times} \otimes \mathbb{Q}_{p} / \mathbb{Z}_{p}\right)^{\chi}$, if $\ell \neq p$,

- $H_{\mathcal{F}_{\text {can }}}^{1}\left(k_{p}, T\right)=H^{1}\left(k_{p}, T\right)$,

$H_{\mathcal{F}_{\text {can }}^{*}}^{1}\left(k_{p}, T^{*}\right)=0$.

Here, $\mathcal{I}_{\mathfrak{q}}$ stands for a fixed inertia group at $\mathfrak{q}$, and $\mathcal{O}_{L, \ell}:=\mathcal{O}_{L} \otimes \mathbb{Z}_{\ell}$ is the sum of the local units inside $L_{\ell}=\bigoplus_{\mathfrak{q} \mid \ell} L_{\mathfrak{q}}$.

Proof. This is proved in [R3, Sections I.6.2-3].

Definition 2.11. We define the classical Selmer structure $\mathcal{F}_{\mathrm{cl}}$ on $T$ (and $\mathcal{F}_{\text {cl }}^{*}$ on $\left.T^{*}\right)$ by setting $\Sigma\left(\mathcal{F}_{\text {cl }}\right)=\Sigma\left(\mathcal{F}_{\text {can }}\right)$, and by letting

- $H_{\mathcal{F}_{\mathrm{cl}}}^{1}\left(k_{\ell}, T\right)=H_{\mathcal{F}_{\text {can }}}^{1}\left(k_{\ell}, T\right)$, and $H_{\mathcal{F}_{\mathrm{cl}}^{*}}^{1}\left(k_{\ell}, T^{*}\right)=H_{\mathcal{F}_{\text {can }}^{*}}^{1}\left(k_{\ell}, T^{*}\right)$, if $\ell \neq p$, 
- $H_{\mathcal{F}_{\mathrm{cl}}}^{1}\left(k_{p}, T\right)=\left(\bigoplus_{\mathfrak{q} \mid p} \operatorname{Hom}\left(G_{L_{\mathfrak{q}}} / \mathcal{I}_{\mathfrak{q}}, \mathbb{Z}_{p}\right)\right)^{\chi^{-1}}$, and $H_{\mathcal{F}_{\mathrm{cl}}^{*}}^{1}\left(k_{p}, T^{*}\right)=\left(\mathcal{O}_{L, p}^{\times} \otimes \mathbb{Q}_{p} / \mathbb{Z}_{p}\right)^{\chi}$.

REMARK 2.12. If we assume that (A1) holds, it follows from the proof of [R3, Proposition III.2.6] (see also [MR1, Lemma 6.1.2]) that $H_{\mathcal{F}_{\mathrm{cl}}}^{1}\left(k_{p}, T\right)=0$ and that $H_{\mathcal{F}_{\mathrm{cl}}^{*}}^{1}\left(k_{p}, T^{*}\right)=H^{1}\left(k_{p}, T^{*}\right)$. We therefore have the following exact sequences:

$$
\begin{aligned}
0 \longrightarrow H_{\mathcal{F}_{\mathrm{cl}}}^{1}(k, T) \longrightarrow H_{\mathcal{F}_{\text {can }}}^{1}(k, T) \stackrel{\operatorname{loc}_{p}}{\longrightarrow} H^{1}\left(k_{p}, T\right), \\
0 \longrightarrow H_{\mathcal{F}_{\text {can }}^{*}}^{1}\left(k, T^{*}\right) \longrightarrow H_{\mathcal{F}_{\text {cl }}^{*}}^{1}\left(k, T^{*}\right) \stackrel{\operatorname{loc}_{p}^{*}}{\longrightarrow} H^{1}\left(k_{p}, T^{*}\right) .
\end{aligned}
$$

Furthermore, the image of $\operatorname{loc}_{p}$ is the orthogonal complement of the image of $\operatorname{loc}_{p}^{*}$, by the Poitou-Tate global duality theorem. We note that the classical Selmer group $H_{\mathcal{F}_{\mathrm{cl}}}^{1}(k, T)$ (resp., $H_{\mathcal{F}_{\mathrm{cl}}^{*}}^{1}\left(k, T^{*}\right)$ ) is denoted by $\mathcal{S}(k, T)$ (resp., by $\left.\mathcal{S}\left(k, W^{*}\right)\right)$ in [R3].

Proposition 2.13. Let $A_{L}$ denote the p-part of the ideal class group of L. Then, $H_{\mathcal{F}_{\mathrm{cl}}}^{1}(k, T)=0$ and $H_{\mathcal{F}_{\mathrm{cl}}^{*}}^{1}\left(k, T^{*}\right) \cong A_{L}^{\chi}$.

Proof. Proposition 6.1.3 of [MR1] gives

$$
H_{\mathcal{F}_{\mathrm{cl}}}^{1}(k, T)={\underset{m}{\lim }}_{\lim } \operatorname{Hom}\left(A_{L}^{\chi}, \mathbb{Z} / p^{m} \mathbb{Z}\right)=\operatorname{Hom}\left(A_{L}^{\chi}, \mathbb{Z}_{p}\right)
$$

we note that the propagation of $\mathcal{F}_{\mathrm{cl}}$ to $\mathbb{Z} / p^{m} \mathbb{Z}(\chi)$ coincides with the Selmer structure $\mathcal{F}^{*}$ of $\left[\mathrm{MR} 1\right.$, Section 6.1]. Since $A_{L}^{\chi}$ is finite, it follows that $H_{\mathcal{F}_{\mathrm{cl}}}^{1}(k, T)=0$.

Similarly, the propagation of $\mathcal{F}_{\text {cl }}^{*}$ to $\boldsymbol{\mu}_{p^{m}} \otimes \chi^{-1}$ coincides with the Selmer structure $\mathcal{F}$ of [MR1, Section 6.1]. It therefore follows from [MR1, Proposition 6.1.3] that there is an exact sequence

$$
0 \longrightarrow\left(\mathcal{O}_{L}^{\times} /\left(\mathcal{O}_{L}^{\times}\right)^{p^{m}}\right)^{\chi} \longrightarrow H_{\mathcal{F}_{\mathrm{cl}}^{*}}^{1}\left(k, T^{*}\left[p^{m}\right]\right) \longrightarrow A_{L}\left[p^{m}\right]^{\chi} \longrightarrow 0 .
$$

Taking the direct limit with respect to $m$, we obtain the following exact sequence:

$$
0 \longrightarrow\left(\mathcal{O}_{L}^{\times} \otimes \mathbb{Q}_{p} / \mathbb{Z}_{p}\right)^{\chi} \longrightarrow H_{\mathcal{F}_{\mathrm{cl}}^{*}}^{1}\left(k, T^{*}\right) \longrightarrow A_{L}^{\chi} \longrightarrow 0 .
$$

Since $\chi$ is totally odd, it follows from [T, proposition I.3.4] that $\left(\mathcal{O}_{L}^{\times}\right)^{\chi}$ is finite; hence, $\left(\mathcal{O}_{L}^{\times} \otimes \mathbb{Q}_{p} / \mathbb{Z}_{p}\right)^{\chi}=0$. This completes the proof of the proposition. 
2.2.2. Selmer groups over $k_{\infty}$. Let $k_{n}$ denote the unique subfield $k_{\infty}$, which is of degree $p^{n}$ over $k$. We also set $L_{n}=L \cdot k_{n}$. Repeating the arguments of the previous section (replacing the totally real field $k$ with the totally real field $k_{n}$ ), we prove the following.

LEMMA 2.14. There is a canonical identification

$$
\underset{n}{\lim } H_{\mathcal{F}_{\mathrm{cl}}}^{1}\left(k_{n}, T^{*}\right)=\underset{n}{\lim } A_{L_{n}}^{\chi} .
$$

\subsection{Modifying the local conditions at $p$}

When the core Selmer rank of a Selmer structure (in the sense of [MR1]; see also Section 2.5 below) is greater than 1, it produces a Selmer group which is difficult to control using the Kolyvagin system machinery of [MR1]. As we will see in Section 2.5, the Selmer structure $\mathcal{F}_{\text {can }}$ on $T$ (resp., $\mathcal{F}_{\Lambda}$ on $T \otimes \Lambda)$ has core Selmer rank $r=[k: \mathbb{Q}]$. Hence, to be able to utilize the Kolyvagin system machinery, we will need to modify $\mathcal{F}_{\text {can }}$ and $\mathcal{F}_{\Lambda}$ appropriately. This is what we do in this section.

Throughout this section we assume (A1) and (A2).

\subsubsection{Local conditions at $p$ over $k$.}

LEMMA 2.15. Under our running hypotheses,

$$
H^{1}\left(k_{p}, T\right):=\bigoplus_{\wp \mid p} H^{1}\left(k_{\wp}, T\right)
$$

is a free $\mathbb{Z}_{p}$-module of rank $r=[k: \mathbb{Q}]$.

Proof. We first prove this using the general structure theory of semilocal cohomology groups at $p$. All the references in this proof are to [B2, Appen$\operatorname{dix} \mathrm{A}$ ] (we note that the results quoted there are originally due to Benois, Colmez, Herr, and Perrin-Riou).

By [B2, Theorem A.8(i)], the $\Lambda$-torsion submodule $H^{1}\left(k_{p}, T \otimes \Lambda\right)_{\text {tors }}$ is isomorphic to $\bigoplus_{\wp \mid p} T^{H_{k_{\wp}}}$, where $H_{k_{\wp}}=\operatorname{Gal}\left(\overline{k_{\wp}} / k_{\wp, \infty}\right)$. Since we assume (A1), it follows that $H^{1}\left(k_{p}, T \otimes \Lambda\right)_{\text {tors }}=0$. Then [B2, Theorem A.8(ii)] concludes that the $\Lambda$-module $H^{1}\left(k_{p}, T \otimes \Lambda\right)$ is free rank $r$. Furthermore,

$$
\operatorname{coker}\left[H^{1}\left(k_{p}, T \otimes \Lambda\right) \longrightarrow H^{1}\left(k_{p}, T\right)\right]=H^{2}\left(k_{p}, T \otimes \Lambda\right)[\gamma-1],
$$

where $\gamma$ is any topological generator of $\Gamma$. However, it follows from [B3, Lemma 2.11] that $H^{2}\left(k_{p}, T \otimes \Lambda\right)=0$; hence, the map

$$
H^{1}\left(k_{p}, T \otimes \Lambda\right) \longrightarrow H^{1}\left(k_{p}, T\right)
$$


is surjective. The lemma now follows.

REMARK 2.16. There is a more direct proof of Lemma 2.15. In this remark, we include this alternative proof of this lemma.

By the explicit description of the semilocal cohomology groups in (2.6),

$$
H^{1}\left(k_{p}, T\right) \cong \bigoplus_{\wp \mid p}\left(\bigoplus_{\mathfrak{q} \mid \wp} \operatorname{Hom}\left(G_{L_{\mathfrak{q}}}, \mathbb{Z}_{p}\right)\right)^{\chi^{-1}}
$$

It follows at once from this description that $H^{1}\left(k_{p}, T\right)$ is $\mathbb{Z}_{p}$-torsion free, hence free. Further, since $\mathbb{Z}_{p}$ is an abelian group, we may rewrite the equality above as

$$
H^{1}\left(k_{p}, T\right) \cong \bigoplus_{\wp \mid p}\left(\bigoplus_{\mathfrak{q} \mid \wp} \operatorname{Hom}\left(G_{L_{\mathfrak{q}}}^{\mathrm{ab}}, \mathbb{Z}_{p}\right)\right)^{\chi^{-1}}
$$

where $G_{L_{\mathfrak{q}}}^{\text {ab }}$ stands for the abelianization of $G_{L_{\mathfrak{q}}}$. By local class field theory, $G_{L_{\mathfrak{q}}}^{\mathrm{ab}} \cong L_{\mathfrak{q}}^{\wedge}$, the $p$-adic completion of the multiplicative group of $L_{\mathfrak{q}}$. Further, the valuation map val $_{\mathfrak{q}}$ gives an isomorphism

$$
L_{\mathfrak{q}}^{\times} \stackrel{\operatorname{val}_{\mathfrak{q}}}{\longrightarrow} \mathbb{Z}_{p} \oplus \mathcal{O}_{L_{\mathfrak{q}}}^{\times, \wedge}
$$

We therefore have

$$
\begin{aligned}
H^{1}\left(k_{p}, T\right) & \cong \operatorname{Hom}\left(\bigoplus_{\mathfrak{q} \mid p}\left(\mathbb{Z}_{p} \oplus \mathcal{O}_{L_{\mathfrak{q}}}^{\times, \wedge}\right), \mathbb{Z}_{p}\right)^{\chi^{-1}} \\
& \cong \operatorname{Hom}\left(\left(\bigoplus_{\mathfrak{q} \mid p} \mathbb{Z}_{p}\right)^{\chi} \oplus\left(\bigoplus_{\mathfrak{q} \mid p} \mathcal{O}_{L_{\mathfrak{q}}}^{\times, \wedge}\right)^{\chi}, \mathbb{Z}_{p}\right)
\end{aligned}
$$

It follows from $(\mathrm{A} 1)$ that $\left(\bigoplus_{\mathfrak{q} \mid p} \mathbb{Z}_{p}\right)^{\chi}=0$; hence,

$$
H^{1}\left(k_{p}, T\right) \cong \operatorname{Hom}\left(\left(\bigoplus_{\mathfrak{q} \mid p} \mathcal{O}_{L_{\mathfrak{q}}}^{\times, \wedge}\right)^{\chi}, \mathbb{Z}_{p}\right)
$$

To prove the lemma, it suffices to check that the $\mathbb{Q}_{p}$-dimension of $V:=$ $\left(\bigoplus_{\mathfrak{q} \mid p} \mathcal{O}_{L_{\mathfrak{q}}}^{\times, \wedge} \otimes \mathbb{Q}_{p}\right)^{\chi}$ is equal to $r$. The $p$-adic logarithm gives a homomorphism $\mathcal{O}_{L_{\mathfrak{q}}}^{\times, \wedge} \rightarrow \mathcal{O}_{L_{\mathfrak{q}}}$ with finite kernel and cokernel. Hence,

$$
V=\left(\bigoplus_{\mathfrak{q} \mid p} \mathcal{O}_{L_{\mathfrak{q}}}^{\times, \wedge} \otimes \mathbb{Q}_{p}\right)^{\chi}=\left(\bigoplus_{\mathfrak{q} \mid p} \mathcal{O}_{L_{\mathfrak{q}}} \otimes \mathbb{Q}_{p}\right)^{\chi}=\left(L \otimes \mathbb{Q}_{p}\right)^{\chi}
$$

and therefore the $\mathbb{Q}_{p}$-dimension of $V$ equals $r$ by the normal basis theorem. 
Definition 2.17. Fix a $\mathbb{Z}_{p}$-direct summand $\mathcal{L} \subset H^{1}\left(k_{p}, T\right)$ such that $\mathcal{L}$ is free of rank 1. Fix also a generator $\varphi=\varphi_{\mathcal{L}}$ of $\mathcal{L}$. Define the $\mathcal{L}$-modified Selmer structure $\mathcal{F}_{\mathcal{L}}$ on $T$ as follows:

- $\Sigma\left(\mathcal{F}_{\mathcal{L}}\right)=\Sigma\left(\mathcal{F}_{\text {can }}\right)$

- if $\lambda \nmid p, H_{\mathcal{F}_{\mathcal{L}}}^{1}\left(k_{\lambda}, T\right)=H_{\mathcal{F}_{\text {can }}}^{1}\left(k_{\lambda}, T\right)$,

- $H_{\mathcal{F}_{\mathcal{L}}}^{1}\left(k_{p}, T\right):=\mathcal{L} \subset H^{1}\left(k_{p}, T\right)=H_{\mathcal{F}_{\text {can }}}^{1}\left(k_{p}, T\right)$.

2.3.2. Local conditions at $p$ over $k_{\infty}$. Set $\Gamma=\operatorname{Gal}\left(k_{\infty} / k\right)$ as before. Let $k_{\wp}$ denote the completion of $k$ at $\wp$, and let $k_{\wp, \infty}$ denote the cyclotomic $\mathbb{Z}_{p^{-}}$ extension of $k_{\wp}$. Since we assume (A2), we may identify $\operatorname{Gal}\left(k_{\wp, \infty} / k_{\wp}\right)$ by $\Gamma$ for each $\wp \mid p$, and henceforth $\Gamma$ will stand for any of these Galois groups. Let $\Lambda=\mathbb{Z}_{p}[[\Gamma]]$ be the cyclotomic Iwasawa algebra, as usual. We also fix a topological generator $\gamma$ of $\Gamma$, and we set $\mathbf{X}=\gamma-1$. We will occasionally identify $\Lambda$ by the power series ring $\mathbb{Z}_{p}[[\mathbf{X}]]$.

LEMMA 2.18. Under the assumptions (A1) and (A2),

$$
H^{1}\left(k_{p}, T \otimes \Lambda\right):=\bigoplus_{\wp \mid p} H^{1}\left(k_{\wp}, T \otimes \Lambda\right)
$$

is a free $\Lambda$-module of rank $r$.

Proof. This is checked in the first part of the proof of Lemma 2.15.

Definition 2.19. Fix a $\Lambda$-rank 1 direct summand $\mathbb{L} \subset H^{1}\left(k_{p}, T \otimes \Lambda\right)$ such that $\mathbb{L}$ maps onto $\mathcal{L}$ under projection

$$
H^{1}\left(k_{p}, T \otimes \Lambda\right) \longrightarrow H^{1}\left(k_{p}, T\right) .
$$

Fix also a generator $\Phi=\Phi_{\mathbb{L}}$ of $\mathbb{L}$, which maps to $\varphi=\varphi_{\mathcal{L}}$ under projection (2.12). Define the $\mathbb{L}$-modified Selmer structure $\mathcal{F}_{\mathbb{L}}$ on $T \otimes \Lambda$ as follows:

- $\Sigma\left(\mathcal{F}_{\mathbb{L}}\right)=\Sigma\left(\mathcal{F}_{\Lambda}\right)$,

- if $\lambda \nmid p, H_{\mathcal{F}_{\mathbb{L}}}^{1}\left(k_{\lambda}, T \otimes \Lambda\right)=H_{\mathcal{F}_{\Lambda}}^{1}\left(k_{\lambda}, T \otimes \Lambda\right)$,

- $H_{\mathcal{F}_{\mathbb{L}}}^{1}\left(k_{p}, T \otimes \Lambda\right):=\mathbb{L} \subset H^{1}\left(k_{p}, T \otimes \Lambda\right)=H_{\mathcal{F}_{\Lambda}}^{1}\left(k_{p}, T \otimes \Lambda\right)$.

REMARK 2.20. By definition, the image of $H_{\mathcal{F}_{\mathbb{L}}}^{1}\left(k_{p}, T \otimes \Lambda\right)$ is $H_{\mathcal{F}_{\mathcal{L}}}^{1}\left(k_{p}, T\right)$ under the map $H^{1}\left(k_{p}, T \otimes \Lambda\right) \rightarrow H^{1}\left(k_{p}, T\right)$. Further, it follows from [MR1, Lemma 5.3.1(ii)] for $\ell \neq p$ that $H_{\mathcal{F}_{\mathbb{L}}}^{1}\left(k_{\ell}, T \otimes \Lambda\right)$ also maps to $H_{\mathcal{F}_{\mathcal{L}}}^{1}\left(k_{\ell}, T\right)$ under the natural map $H^{1}\left(k_{\ell}, T \otimes \Lambda\right) \rightarrow H^{1}\left(k_{\ell}, T\right)$. In other words, $\mathcal{F}_{\mathbb{L}}$ propagates to $\mathcal{F}_{\mathcal{L}}$, and there is an induced map

$$
H_{\mathcal{F}_{\mathbb{L}}}^{1}(k, T \otimes \Lambda) \longrightarrow H_{\mathcal{F}_{\mathcal{L}}}^{1}(k, T)
$$




\subsection{Global duality and a comparison of Selmer groups}

In this section, we compare the classical Selmer group (which we wish to relate to $L$-values) to the modified Selmer groups (for which we will apply the Kolyvagin system machinery and which we will compute in terms of $L$-values). The necessary tool to accomplish this comparison is Poitou-Tate global duality.

2.4.1. Comparison over $k$. The definition of the modified Selmer structure $\mathcal{F}_{\mathcal{L}}$ and Remark 2.12 give us the following exact sequences:

$$
\begin{aligned}
0 \longrightarrow H_{\mathcal{F}_{\mathrm{cl}}}^{1}(k, T) \longrightarrow H_{\mathcal{F}_{\mathcal{L}}}^{1}(k, T) \stackrel{\operatorname{loc}_{p}}{\longrightarrow} \mathcal{L}, \\
0 \longrightarrow H_{\mathcal{F}_{\mathcal{L}}^{*}}^{1}\left(k, T^{*}\right) \longrightarrow H_{\mathcal{F}_{\mathrm{cl}}^{*}}^{1}\left(k, T^{*}\right) \stackrel{\operatorname{loc}_{p}^{*}}{\longrightarrow} \frac{H_{\mathcal{F}_{\mathrm{cl}}^{*}}^{1}\left(k_{p}, T^{*}\right)}{H_{\mathcal{F}_{\mathcal{L}}^{*}}^{1}\left(k_{p}, T^{*}\right)}
\end{aligned}
$$

Poitou-Tate global duality (see [R3, Theorem I.7.3], [Mi, Theorem I.4.10]) states that the image of $\operatorname{loc}_{p}$ is the orthogonal complement of the image of $\operatorname{loc}_{p}^{*}$ with respect to the local Tate pairing. Using this fact, together with Proposition 2.13, one may prove the following proposition for $T=\mathbb{Z}_{p}(\chi)$ as above. Note that $H_{\mathcal{F}_{\mathrm{cl}}}^{1}(k, T)=0$ by Proposition 2.13. See [R3, Theorem I.7.3] for further details.

Proposition 2.21. We have an exact sequence

$$
0 \rightarrow H_{\mathcal{F}_{\mathcal{L}}}^{1}(k, T) \stackrel{\operatorname{loc}_{p}}{\longrightarrow} \mathcal{L} \stackrel{\left(\operatorname{loc}_{p}^{*}\right)^{\vee}}{\longrightarrow}\left(H_{\mathcal{F}_{\mathrm{cl}}^{*}}^{1}\left(k, T^{*}\right)\right)^{\vee} \longrightarrow\left(H_{\mathcal{F}_{\mathcal{L}}^{*}}^{1}\left(k, T^{*}\right)\right)^{\vee} \rightarrow 0
$$

where the map $\left(\operatorname{loc}_{p}^{*}\right)^{\vee}$ is induced from localization at $p$ and the local Tate pairing between $H^{1}\left(k_{p}, T\right)$ and $H^{1}\left(k_{p}, T^{*}\right)$.

Suppose that $c \in H_{\mathcal{F}_{\mathcal{L}}}^{1}(k, T)$ is any class. We still write $c$ for the image of the class $c$ inside $\mathcal{L}=H_{\mathcal{F}_{\mathcal{L}}}^{1}\left(k_{p}, T\right)$ under the (injective) map $\operatorname{loc}_{p}$.

COROLlary 2.22. The following sequence is exact:

$$
0 \rightarrow \frac{H_{\mathcal{F}_{\mathcal{L}}}^{1}(k, T)}{\mathbb{Z}_{p} \cdot c} \stackrel{\operatorname{loc}_{p}}{\longrightarrow} \frac{\mathcal{L}}{\mathbb{Z}_{p} \cdot c} \stackrel{\left(\operatorname{loc}_{p}^{*}\right)^{\vee}}{\longrightarrow}\left(H_{\mathcal{F}_{\mathrm{cl}}^{*}}^{1}\left(k, T^{*}\right)\right)^{\vee} \rightarrow\left(H_{\mathcal{F}_{\mathcal{L}}^{*}}^{1}\left(k, T^{*}\right)\right)^{\vee} \rightarrow 0 .
$$


2.4.2. Comparison over $k_{\infty}$. Repeating the argument of Proposition 2.21 for each field $k_{n}$ (instead of $k$ ) and passing to the inverse limit, we obtain the following.

Proposition 2.23. Both of the following sequences of $\Lambda$-modules are exact.

(i) $0 \rightarrow H_{\mathcal{F}_{\mathbb{L}}}^{1}(k, T \otimes \Lambda) \stackrel{\operatorname{loc}_{p}}{\longrightarrow} \mathbb{L} \longrightarrow\left(\lim _{\longrightarrow} A_{L_{n}}^{\chi}\right)^{\vee} \longrightarrow\left(H_{\mathcal{F}_{\mathbb{L}}^{*}}^{1}\left(k,(T \otimes \Lambda)^{*}\right)\right)^{\vee} \rightarrow 0$.

(ii) For any class $c \in H^{1}(k, T \otimes \Lambda)$,

$0 \rightarrow \frac{H_{\mathcal{F}_{\mathbb{L}}}^{1}(k, T \otimes \Lambda)}{\Lambda \cdot c} \stackrel{\operatorname{loc}_{p}}{\longrightarrow} \frac{\mathbb{L}}{\Lambda \cdot c} \longrightarrow\left(\underset{\lim }{\longrightarrow} A_{L_{n}}^{\chi}\right)^{\vee} \longrightarrow\left(H_{\mathcal{F}_{\mathbb{L}}^{*}}^{1}\left(k,(T \otimes \Lambda)^{*}\right)\right)^{\vee} \longrightarrow 0$.

Proof. We give only a sketch since similar versions of this proposition are already available in the literature (see [R3, Theorems I.7.3, III.2.10], [dS, Section III.1.7]).

Thanks to the argument of Proposition 2.21 and [R3, Proposition B.1.1], there is an exact sequence

$$
\begin{aligned}
0 \longrightarrow & \underset{n}{\lim } H_{\mathcal{F}_{\mathcal{L}_{n}}}^{1}\left(k_{n}, T\right) \longrightarrow \underset{n}{\longrightarrow} \lim _{n} \mathcal{L}_{n} \longrightarrow\left(\underset{n}{\longrightarrow} H_{\mathcal{F}_{\mathrm{cl}}^{*}}^{1}\left(k_{n}, T^{*}\right)\right)^{\vee} \\
\longrightarrow & \left.\underset{n}{\lim } H_{\mathcal{F}_{\mathcal{L}_{n}}^{*}}^{1}\left(k_{n}, T^{*}\right)\right)^{\vee} \longrightarrow 0,
\end{aligned}
$$

where $\mathcal{L}_{n}$ is the image of $\mathbb{L}$ under the natural map

$$
H^{1}\left(k_{p}, T \otimes \Lambda\right) \longrightarrow H^{1}\left(\left(k_{n}\right)_{p}, T\right)
$$

By definition, $\lim _{n} \mathcal{L}_{n}=\mathbb{L}$, and by [MR1, Lemma 5.3.1] (or rather by its proof), it follows that there is a canonical isomorphism

$$
\lim _{n} H_{\mathcal{F}_{\mathcal{L}_{n}}}^{1}\left(k_{n}, T\right) \cong H_{\mathcal{F}_{\mathbb{L}}}^{1}(k, T \otimes \Lambda)
$$

Furthermore, by Lemma 2.14, $\lim _{\longrightarrow} H_{\mathcal{F}_{\mathrm{cl}}^{*}}^{1}\left(k_{n}, T^{*}\right)=\lim _{\longrightarrow} A_{L_{n}}^{\chi}$. Finally, by Shapiro's lemma,

$$
H^{1}\left(k_{n}, T^{*}\right)=H^{1}\left(k, T^{*} \otimes \mathbb{Z}_{p}\left[\Gamma_{n}\right]\right),
$$

where $\Gamma_{n}=\operatorname{Gal}\left(k_{n} / k\right)$; hence,

$$
\underset{n}{\lim } H^{1}\left(k_{n}, T^{*}\right)=H^{1}\left(k, \underset{n}{\lim } T^{*} \otimes \mathbb{Z}_{p}\left[\Gamma_{n}\right]\right) .
$$


Now, using the fact that the functors $-\otimes_{\mathbb{Z}_{p}} \mathbb{Z}_{p}\left[\Gamma_{n}\right]$ and $\operatorname{Hom}_{\mathbb{Z}_{p}}\left(\mathbb{Z}_{p}\left[\Gamma_{n}\right],-\right)$ are adjoint functors (we drop the subscripts below and write $\otimes$ and Hom for short), it follows that

$$
\begin{aligned}
(T \otimes \Lambda)^{*} & :=\operatorname{Hom}\left({\underset{n}{n}}_{\lim } T \otimes \mathbb{Z}_{p}\left[\Gamma_{n}\right], \mathbb{Q}_{p} / \mathbb{Z}_{p}\right)(1) \\
& \cong \underset{n}{\lim } \operatorname{Hom}\left(T, \operatorname{Hom}\left(\mathbb{Z}_{p}\left[\Gamma_{n}\right], \mathbb{Q}_{p} / \mathbb{Z}_{p}\right)\right)(1) \\
& \cong \underset{n}{\lim } \operatorname{Hom}\left(T, \mathbb{Q}_{p} / \mathbb{Z}_{p}\left[\Gamma_{n}\right]\right)(1) \\
& \cong \underset{n}{\lim } \operatorname{Hom}\left(T, \mathbb{Q}_{p} / \mathbb{Z}_{p}\right)(1) \otimes \mathbb{Z}_{p}\left[\Gamma_{n}\right]=: \underset{n}{\lim } T^{*} \otimes \mathbb{Z}_{p}\left[\Gamma_{n}\right],
\end{aligned}
$$

where the isomorphism of the modules in the second and the third lines comes from the isomorphism

$$
\begin{aligned}
\operatorname{Hom}\left(\mathbb{Z}_{p}\left[\Gamma_{n}\right], \mathbb{Q}_{p} / \mathbb{Z}_{p}\right) \stackrel{\sim}{\longrightarrow} \mathbb{Q}_{p} / \mathbb{Z}_{p}\left[\Gamma_{n}\right] \\
f \longmapsto \sum_{\gamma \in \Gamma_{n}} f(\gamma) \cdot \gamma
\end{aligned}
$$

of $\mathbb{Z}_{p}\left[\Gamma_{n}\right]$-modules. This and (2.13) (together with its semilocal analogue) show at once that

$$
\underset{n}{\lim } H_{\mathcal{F}_{\mathcal{L}_{n}}^{*}}^{1}\left(k_{n}, T^{*}\right)=H_{\mathcal{F}_{\mathbb{L}}^{*}}^{1}\left(k,(T \otimes \Lambda)^{*}\right) .
$$

This completes the proof of (i), and (ii) follows trivially from (i).

\subsection{Kolyvagin systems for modified Selmer groups}

This section closely follows the exposition of [B1, Section 1.2] and [B2, Section 2.5]. We assume (A1) and (A2) throughout this section.

REMARK 2.24. It is straightforward to verify that the following hypotheses (which were introduced in [MR1, Section 3.5]) hold for $T=\mathbb{Z}_{p}(\chi)$.

(H.1) The residual $\mathbb{F}_{p}\left[\left[G_{k}\right]\right]$-representation $T / p T$ is absolutely irreducible.

(H.2) There is a $\tau \in G_{k}$ such that $\tau=1$ on $\mu_{p^{\infty}}$ and $T /(\tau-1) T$ is free of rank 1 over $\mathbb{Z}_{p}$.

(H.3) $H^{0}(k, T / p T)=H^{0}\left(k, T^{*}[p]\right)=0$.

(H.4) $\operatorname{Hom}_{\mathbb{F}_{p}\left[\left[G_{k}\right]\right]}\left(T / p T, T^{*}[p]\right)=0$.

We remark that hypothesis (H.3) above is implied by what Mazur and Rubin call (H.3) (see [MR1, Lemma 3.5.2]). Hypothesis (H.3) is sufficient for our purposes. 
Let $\mathcal{P}$ denote the set whose elements are prime ideals of $k$ which are prime to $p \mathfrak{f}_{\chi}$. For each positive integer $m$ and $n$, let

$$
\mathcal{P}_{m+n}=\left\{\mathfrak{q} \in \mathcal{P}: \mathfrak{q} \text { splits completely in } L\left(\mu_{p^{m+n+1}}\right) / k\right\}
$$

be a subset of $\mathcal{P}$. Note that $\mathcal{P}_{m+n}$ is exactly the set of primes determined by [R3, Definition IV.1.1] when $T=\mathbb{Z}_{p}(\chi)$. Hypothesis (H.5) of [MR1, Section 3.5] holds with this choice of $\mathcal{P}$. Let $\mathcal{N}=\mathcal{N}(\mathcal{P})$ (resp., $\mathcal{N}_{j}=\mathcal{N}\left(\mathcal{P}_{j}\right) \subset$ $\mathcal{N}$ ) be the square-free products of primes $\mathfrak{q} \in \mathcal{P}$ (resp., in $\mathcal{P}_{j}$ ), with the convention that $1 \in \mathcal{N}_{j} \subset \mathcal{N}$.

Using [MR1, Lemma 3.7.1], one may also check that $\mathcal{F}_{\text {can }}$ and $\mathcal{F}_{\mathcal{L}}$ satisfy hypothesis (H.6) of [MR1, Section 3.5]. We may therefore apply the main results of [MR1]. In particular, the existence of Kolyvagin systems for these Selmer structures will be decided by their core Selmer ranks (for a definition, see [MR1, Definitions 4.1.11 and 5.2.4]). Let $\mathcal{X}(T, \mathcal{F})$ denote the core Selmer rank of the Selmer structure $\mathcal{F}$, for $\mathcal{F}=\mathcal{F}_{\text {can }}$ or for $\mathcal{F}=\mathcal{F}_{\mathcal{L}}$.

Proposition 2.25. We have $\mathcal{X}\left(T, \mathcal{F}_{\text {can }}\right)=r(=[k: \mathbb{Q}])$.

Proof. This follows from [MR1, Theorem 5.2.15], applied with the base field $k$ (instead of $\mathbb{Q}$; we therefore have $r$ real places instead of one) and using our assumption that $\chi$ is totally odd.

Proposition 2.26. The core Selmer rank $\mathcal{X}\left(T, \mathcal{F}_{\mathcal{L}}\right)$ of the Selmer structure $\mathcal{F}_{\mathcal{L}}$ on $T$ is one.

Proof. The proof of this proposition is identical to the proof of [B1, Proposition 1.8].

2.5.1. Kolyvagin systems over $k$. We recall the definition of the (generalized) module of Kolyvagin systems (introduced in [MR1]) for the Selmer triple $\left(T, \mathcal{F}_{\mathcal{L}}, \mathcal{P}\right)$.

Definition 2.27 (compare [MR1, Definition 3.1.6]). Define the (generalized) module of Kolyvagin systems

$$
\overline{\mathbf{K S}}\left(T, \mathcal{F}_{\mathcal{L}}, \mathcal{P}\right):=\varliminf_{s} \underset{j}{\lim } \lim \mathbf{K}\left(T / p^{s} T, \mathcal{F}_{\mathcal{L}}, \mathcal{P}_{j}\right)
$$

where $\mathbf{K S}\left(T / p^{s} T, \mathcal{F}_{\mathcal{L}}, \mathcal{P}_{j}\right)$ is the module of Kolyvagin systems for the Selmer structure $\mathcal{F}_{\mathcal{L}}$ on the representation $T / p^{s} T$, as in [MR1, Definition 3.1.3].

We call an element of $\overline{\mathbf{K S}}\left(T, \mathcal{F}_{\mathcal{L}}, \mathcal{P}\right)$ an $\mathcal{L}$-restricted Kolyvagin system for $T$. 
REMARK 2.28. In order to define Kolyvagin systems, one first needs to define the "transverse local condition" (see [MR1, Definition 1.1.6(iv)]). In this remark, we briefly recall this definition. Let $F$ be any local field, and fix once and for all an abelian extension $F^{\prime} / F$ which is totally and tamely ramified and, moreover, is a maximal such extension. When $F=\mathbb{Q}_{\ell}$, then there is a natural choice for $F^{\prime}$, namely, $F^{\prime}=\mathbb{Q}_{\ell}\left(\boldsymbol{\mu}_{\ell}\right)$. In general, we simply fix an extension $F^{\prime}$ as above and define the transverse local condition to be

$$
H_{\mathrm{tr}}^{1}(F, X)=\operatorname{ker}\left\{H^{1}(F, X) \longrightarrow H^{1}\left(F^{\prime}, X\right)\right\},
$$

for appropriate quotients $X$ of $T$.

Let $\mathfrak{q} \in \mathcal{P}_{j}$ for some $j$, and consider now the case $F=k_{\mathfrak{q}}$. Starting from Section 4 , we will insist that the extension $F^{\prime}$ contains $k(\mathfrak{q})_{\mathfrak{q}}$, where $k(\mathfrak{q})$ is the maximal $p$-extension inside the ray class field of $k$ modulo the prime ideal $\mathfrak{q}$. Although we do not need this assumption for the results in Section 2.5, it is necessary to choose $F^{\prime}$ in this manner to be able to modify the arguments of [MR1, Theorem 3.2.4] in order to obtain a proof of Theorem 4.1 below.

Proposition 2.29. The $\mathbb{Z}_{p}$-module $\overline{\mathbf{K S}}\left(T, \mathcal{F}_{\mathcal{L}}, \mathcal{P}\right)$ is free of rank 1 . Furthermore, it is generated by a Kolyvagin system $\kappa \in \overline{\mathbf{K S}}\left(T, \mathcal{F}_{\mathcal{L}}, \mathcal{P}\right)$ whose image (under the canonical map induced from reduction mod $p$ ) inside $\mathbf{K S}\left(T / p T, \mathcal{F}_{\mathcal{L}}, \mathcal{P}\right)$ is nonzero.

A generator of the cyclic module $\overline{\mathbf{K S}}\left(T, \mathcal{F}_{\mathcal{L}}, \mathcal{P}\right)$ will be called a primitive Kolyvagin system.

Proof. This is immediate after Proposition 2.26 and [MR1, Theorem 5.2.10]. To apply [MR1, Theorem 5.2.10], one needs to verify that [MR1, Section 3.5, hypotheses (H.1)-(H.6)] hold true for the triple $\left(T, \mathcal{F}_{\mathcal{L}}, \mathcal{P}\right)$.

Remark 2.30. Using Proposition 2.26 and [MR1, Proposition 5.2.9], the generalized module of Kolyvagin systems $\overline{\mathbf{K S}}\left(T, \mathcal{F}_{\mathcal{L}}, \mathcal{P}\right)$ may be identified by the module of Kolyvagin systems $\mathbf{K S}\left(T, \mathcal{F}_{\mathcal{L}}, \mathcal{P}\right)$ (defined as in [MR1, Definition 3.1.3]). We will use this identification without warning.

We record here the main application of a Kolyvagin system for the Selmer triple $\left(T, \mathcal{F}_{\mathcal{L}}, \mathcal{P}\right)$. Suppose that $\left\{\left\{\kappa_{\tau}(s)\right\}_{\tau \in \mathcal{N}_{s}}\right\}_{s}=\kappa \in \overline{\mathbf{K S}}\left(T, \mathcal{F}_{\mathcal{L}}, \mathcal{P}\right)$ is any Kolyvagin system. See [MR1, Section 3] for an explanation of our notation. We loosely say here that $\kappa_{\tau}(s) \in H^{1}\left(k, T / p^{s} T\right)$, and by definition, there is a well-defined element

$$
\kappa_{1}=\left\{\kappa_{1}(s)\right\}_{s} \in{\underset{\varliminf}{s}}_{\lim _{s}} H_{\mathcal{F}_{\mathcal{L}}}^{1}\left(k, T / p^{s} T\right)=H_{\mathcal{F}_{\mathcal{L}}}^{1}(k, T) .
$$


THEOREM 2.31 ([MR1, Theorems 5.2.13, 5.2.14]). Under our running hypotheses,

(i) length $\left(H_{\mathcal{F}_{\mathcal{L}}^{*}}^{1}\left(k, T^{*}\right)\right) \leq \operatorname{length}\left(H_{\mathcal{F}_{\mathcal{L}}}^{1}(k, T) / \mathbb{Z}_{p} \cdot \kappa_{1}\right)$,

(ii) the inequality in (i) is an equality if and only if $\kappa$ is primitive.

REMARK 2.32. Note that the choice of a rank 1 direct summand $\mathcal{L} \subset$ $H^{1}\left(k_{p}, T\right)$ makes our approach somewhat unnatural. We address this issue in this remark. Put

$$
H^{1}\left(k_{p}, T\right)=\bigoplus_{i=1}^{r} \mathcal{L}_{i}
$$

(where each $\mathcal{L}_{i}$ is a free $\mathbb{Z}_{p}$-submodule of $H^{1}\left(k_{p}, T\right)$ of rank 1 ), and consider

$$
\sum_{i=1}^{r} \mathbf{K S}\left(T, \mathcal{F}_{\mathcal{L}_{i}}, \mathcal{P}\right) \subset \mathbf{K S}\left(T, \mathcal{F}_{\text {can }}, \mathcal{P}\right) .
$$

Claim. The sum in (2.15) is a direct sum.

Proof. Assume the contrary: suppose that $0 \neq \boldsymbol{\kappa}^{i} \in \mathbf{K} \mathbf{S}\left(T, \mathcal{F}_{\mathcal{L}_{i}}, \mathcal{P}\right.$ ) (for $i=1, \ldots, r)$ is such that

$$
\sum_{i=1}^{r} a_{i} \kappa^{i}=0
$$

for some $a_{i} \in \mathbb{Z}_{p}$ and $a_{i_{0}} \neq 0$ for a certain $1 \leq i_{0} \leq r$. This means that

$$
a_{i_{0}} \boldsymbol{\kappa}^{i_{0}}=-\sum_{\substack{i=1 \\ i \neq i_{0}}}^{r} a_{i} \boldsymbol{\kappa}^{i} \in \sum_{\substack{i=1 \\ i \neq i_{0}}}^{r} \mathbf{K S}\left(T, \mathcal{F}_{\mathcal{L}_{i}}, \mathcal{P}\right)
$$

Write $\boldsymbol{\kappa}^{i_{0}}=\left\{\kappa_{n}^{i_{0}}\right\}$ (see [MR1, Section 3] for a precise definition of a Kolyvagin system to clarify this notation; see also Remark 2.28 below). Equation (2.16) therefore shows that

$$
\operatorname{loc}_{p}\left(a_{i_{0}} \kappa_{1}^{i_{0}}\right) \in \bigoplus_{\substack{i=1 \\ i \neq i_{0}}}^{r} \mathcal{L}_{i}
$$

Also, by definition, $\operatorname{loc}_{p}\left(a_{i_{0}} \kappa_{1}^{i_{0}}\right) \in \mathcal{L}_{i_{0}}$; using this together with $(2.17)$, we conclude that $\operatorname{loc}_{p}\left(a_{i_{0}} \kappa_{1}^{i_{0}}\right)=0$. The injectivity of $\operatorname{loc}_{p}$ (which we checked in Section 2.4.1) gives $a_{i_{0}} \kappa_{1}^{i_{0}}=0$. 
On the other hand, Proposition 2.21 (applied with $\mathcal{L}=\mathcal{L}_{i_{0}}$ ) shows that $H_{\mathcal{F}_{\mathcal{L}_{i_{0}}}^{*}}^{1}\left(k, T^{*}\right)$ is finite (as the finite group $H_{\mathcal{F}_{\mathrm{cl}}^{*}}^{1}\left(k, T^{*}\right)^{\vee}=\left(A_{L}^{\chi}\right)^{\vee}$ surjects onto its Pontryagin dual). This in turn shows, using [MR1, Theorem 5.2.12(v)], that for any $0 \neq \boldsymbol{\kappa}=\left\{\kappa_{n}\right\} \in \mathbf{K S}\left(T, \mathcal{F}_{\mathcal{L}_{i_{0}}}, \mathcal{P}\right)$, we have $\kappa_{1} \neq 0$. Therefore, $a_{i_{0}} \kappa_{1}^{i_{0}}=0$ implies that $a_{i_{0}} \kappa^{i_{0}}=0$, a contradiction.

Note that, in order to prove the claim above, we used the facts that $\operatorname{loc}_{p}$ is injective (on $H_{\mathcal{F}_{\mathcal{L}_{i}}}^{1}(k, T)$ ) and that $H_{\mathcal{F}_{\mathcal{L}_{i_{0}}^{*}}}^{1}\left(k, T^{*}\right)$ is finite in our current setting. With a bit more work, it is possible to prove this claim without having either of these conditions. We leave the more general proof aside not to digress from the main point of our paper any further.

It would be very interesting to have an answer for the following.

Question: Is the direct sum

$$
\bigoplus_{i=1}^{r} \mathbf{K} \mathbf{S}\left(T, \mathcal{F}_{\mathcal{L}_{i}}, \mathcal{P}\right) \subset \mathbf{K} \mathbf{S}\left(T, \mathcal{F}_{\text {can }}, \mathcal{P}\right)
$$

independent of the choice of decomposition (2.14)?

When the answer to this question is affirmative, we would have a canonically defined rank $r$ submodule of $\mathbf{K S}\left(T, \mathcal{F}_{\text {can }}, \mathcal{P}\right)$. It would be even more tempting to inquire whether this rank $r$ submodule descends from Euler systems. In Section 3 below, we construct a rank $r$ submodule of $\mathbf{K S}\left(T, \mathcal{F}_{\text {can }}, \mathcal{P}\right)$ out of Stickelberger elements, which still does depend on decomposition (2.14).

These questions seem to be out of reach in the current state of the art. We may, however, prove the following weaker (yet still interesting) statement. First, we recall some terminology from [MR1].

Define the module of $L$-values

$$
\begin{aligned}
\mathbf{L V} & =\mathbf{L V}\left(T ;\left\{\mathcal{L}_{i}\right\}_{i=1}^{r}\right) \\
& :=\operatorname{span}_{\mathbb{Z}_{p}}\left(\kappa_{1}: \boldsymbol{\kappa} \in \mathbf{K S}\left(T, \mathcal{F}_{\mathcal{L}_{i}}, \mathcal{P}\right) \text { for some } i\right) \subset H_{\mathcal{F}_{\text {can }}}^{1}(k, T)
\end{aligned}
$$

(Compare this definition with [MR1, Definition 3.1.5].) Note that the $\mathbb{Z}_{p^{-}}$ module $\mathbf{L V}$ depends a priori on the choice of decomposition (2.14).

THEOREM 2.33. The module of L-values $\mathbf{L V}$ is independent of the choice of decomposition (2.14). 
Proof. Fix any generator $\ell_{i}$ of the free $\mathbb{Z}_{p}$-module $\mathcal{L}_{i}$ of rank 1 . Suppose that $\mathcal{L} \subset H^{1}\left(k_{p}, T\right)$ is any rank 1 direct summand (not necessarily one of $\mathcal{L}_{i}$ which appear in (2.14)). Let $\kappa^{\mathcal{L}}=\left\{\kappa_{n}^{\mathcal{L}}\right\}$ be any generator of the cyclic $\mathbb{Z}_{p}$-module $\mathbf{K S}\left(T, \mathcal{F}_{\mathcal{L}}, \mathcal{P}\right)$. To prove the theorem, it suffices to show that $\kappa_{1}^{\mathcal{L}} \in \mathbf{L V}$. We may write

$$
\operatorname{loc}_{p}\left(\kappa_{1}^{\mathcal{L}}\right)=\sum_{i=1}^{r} a_{i} \ell_{i}
$$

with $a_{i} \in \mathbb{Z}_{p}$.

Claim. Let $a_{i}$ be as above. Then

$$
\operatorname{ord}_{p}\left(a_{i}\right) \geq \operatorname{ord}_{p}\left(\# H_{\mathcal{F}_{\mathrm{cl}}^{*}}^{1}\left(k, T^{*}\right)\right)
$$

for all $1 \leq i \leq r$.

Proof of the claim. Let $d=\operatorname{gcd}\left(a_{1}, \ldots, a_{r}\right)$, and set $\alpha_{i}:=\frac{a_{i}}{d} \in \mathbb{Z}_{p}$. By definition, at least one of the $\alpha_{i}$ is a $p$-adic unit. We also set

$$
x:=\operatorname{loc}_{p}\left(\kappa_{1}^{\mathcal{L}}\right)=\sum_{i}^{r} a_{i} \ell_{i} \quad \text { and } \quad y=\frac{x}{d}=\sum_{i}^{r} \alpha_{i} \ell_{i} .
$$

(1) Since $d \cdot y=x \in \mathcal{L}$ and since $H^{1}\left(k_{p}, T\right) / \mathcal{L}$ is $\mathbb{Z}_{p}$-torsion free, it follows that $y \in \mathcal{L}$.

(2) $H^{1}\left(k_{p}, T\right) / \mathbb{Z}_{p} y$ is $\mathbb{Z}_{p}$-torsion free; indeed, suppose that

$$
z=\sum_{i}^{r} \beta_{i} \ell_{i} \in \bigoplus_{i}^{r} \mathcal{L}_{i}=H^{1}\left(k_{p}, T\right)
$$

is such that $m \cdot z \in \mathbb{Z}_{p} y$ for some $m \in \mathbb{Z}_{p}$. This means that

$$
\sum_{i}^{r} m \beta_{i} \ell_{i}=s y=\sum_{i}^{r} s \alpha_{i} \ell_{i}
$$

for some $s \in \mathbb{Z}_{p}$; hence, $m \beta_{i}=s \alpha_{i}$, in particular, $m \mid s \alpha_{i}$ for every $1 \leq$ $i \leq r$. Since $\operatorname{gcd}\left(\alpha_{1}, \ldots, \alpha_{r}\right)=1$, it follows that $m \mid s$; hence, $z=(s / m) \times$ $y \in \mathbb{Z}_{p} y$.

(3) Items (1) and (2) together show that $\mathcal{L}=\mathbb{Z}_{p} y$. 
We may now conclude that

$$
\# \mathcal{L} / \mathbb{Z}_{p} \operatorname{loc}_{p}\left(\kappa_{1}^{\mathcal{L}}\right)=\#\left(\mathbb{Z}_{p} y / \mathbb{Z}_{p} x\right)=p^{\operatorname{ord}_{p}(d)},
$$

with $d$ as above. On the other hand, Corollary 2.22 shows that

$$
\# \frac{\mathcal{L}}{\mathbb{Z}_{p} \operatorname{loc}_{p}\left(\kappa_{1}^{\mathcal{L}}\right)} \geq \# H_{\mathcal{F}_{\mathrm{cl}}^{*}}^{1}\left(k, T^{*}\right) \Longleftrightarrow \# \frac{H_{\mathcal{F}_{\mathcal{L}}}^{1}(k, T)}{\mathbb{Z}_{p} \kappa_{1}^{\mathcal{L}}} \geq \# H_{\mathcal{F}_{\mathcal{L}}^{*}}^{1}\left(k, T^{*}\right) .
$$

The latter statement is the main application of the Kolyvagin system $\kappa^{\mathcal{L}}$ (see Theorem 2.31 above). We therefore conclude that

$$
p^{\operatorname{ord}_{p}(d)} \geq \# H_{\mathcal{F}_{\mathrm{cl}}^{*}}^{1}\left(k, T^{*}\right),
$$

which is our claim.

We now prove that Theorem 2.33 follows from this claim. As in the final paragraph of the proof of the claim above, it follows from Corollary 2.22 and [MR1, Theorems 5.2.10, 5.2.14] that there exists a Kolyvagin system $\tilde{\boldsymbol{\kappa}}^{i} \in \mathbf{K S}\left(T, \mathcal{F}_{\mathcal{L}_{i}}, \mathcal{P}\right)$ such that

$$
\operatorname{loc}_{p}\left(\tilde{\kappa}_{1}^{i}\right)=\# H_{\mathcal{F}_{\mathrm{cl}}^{*}}^{1}\left(k, T^{*}\right) \cdot \ell_{i} \in \mathcal{L}_{i}
$$

for every $i=1, \ldots, r$. By the claim above, there is a Kolyvagin system $\boldsymbol{\kappa}^{i} \in$ $\mathbf{K S}\left(T, \mathcal{F}_{\mathcal{L}_{i}}, \mathcal{P}\right)$ such that $\operatorname{loc}_{p}\left(\kappa_{1}^{i}\right)=a_{i} \ell_{i}\left(\right.$ just set $\left.\boldsymbol{\kappa}^{i}=\left(a_{i} /\left(\# H_{\mathcal{F}_{\mathrm{cl}}^{*}}^{1}\left(k, T^{*}\right)\right)\right) \tilde{\boldsymbol{\kappa}}^{i}\right)$. We therefore have

$$
\operatorname{loc}_{p}\left(\kappa_{1}^{\mathcal{L}}\right)=\sum_{i}^{r} \operatorname{loc}_{p}\left(\kappa_{1}^{i}\right)
$$

and since the map $\operatorname{loc}_{p}$ is injective in our setting, it follows that

$$
\kappa_{1}^{\mathcal{L}}=\sum_{i}^{r} \kappa_{1}^{i} \in \mathbf{L V}
$$

as desired.

We close our remark noting that all this discussion applies equally well in the setting of [B1] and [B2] as long as we assume Leopoldt's conjecture (i.e., the injectivity of $\operatorname{loc}_{p}$ in the setting of [B1] and [B2]). 
2.5.2. Kolyvagin systems over $k_{\infty}$. We start with the observation that the following versions of hypotheses $\mathbb{H}$.T and $\mathbb{H} . \mathbf{s E Z}$ of [B3, Section 2.2] hold for $T$.

$\left(\mathbb{H} . \mathbf{T}_{/ k}\right)\left(T \otimes \mathbb{Q}_{p} / \mathbb{Z}_{p}\right)^{\mathcal{I}_{\lambda}}$ is divisible for every prime $\lambda \nmid p, \lambda \subset k$. $\left(\mathbb{H} . \mathbf{s E Z} \mathbf{H}_{k}\right) H^{0}\left(k_{\wp}, T^{*}\right)=0$ for primes $\wp \mid p$.

We define a Selmer structure $\mathcal{F}_{\text {can }}^{\Lambda}$ on certain quotients of $T \otimes \Lambda$. The following is the Selmer structure $\mathcal{F}_{\text {can }}$ of [B3, Definition 2.2].

Definition 2.34. Suppose $f \in \Lambda$ is any distinguished polynomial, in the sense that the quotient $\Lambda /(f)$ is a free $\mathbb{Z}_{p}$-module of finite rank. Let $\mathcal{F}_{\text {can }}^{\Lambda}$ be the following Selmer structure on $T_{f}:=T \otimes \Lambda /(f)$.

- $\Sigma\left(\mathcal{F}_{\text {can }}^{\Lambda}\right)=\Sigma\left(\mathcal{F}_{\Lambda}\right)$.

- The local conditions are given by

$$
H_{\mathcal{F}_{\text {can }}^{\Lambda}}^{1}\left(k_{\lambda}, T_{f}\right)= \begin{cases}H^{1}\left(k_{\lambda}, T_{f}\right) & \text { if } \lambda \mid p, \\ H_{\mathrm{f}}^{1}\left(k_{\lambda}, T_{f}\right) & \text { if } \lambda \in \Sigma\left(\mathcal{F}_{\text {can }}\right) \text { and } \lambda \nmid p,\end{cases}
$$

with

$$
H_{\mathrm{f}}^{1}\left(k_{\lambda}, T_{f}\right)=\operatorname{ker}\left\{H^{1}\left(k_{\lambda}, T_{f}\right) \longrightarrow H^{1}\left(k_{\lambda}^{\mathrm{unr}}, T_{f} \otimes \mathbb{Q}_{p}\right)\right\},
$$

where $k_{\lambda}^{\text {unr }}$ is the maximal unramified extension of $k_{\lambda}$.

The induced Selmer structure on the quotients $T \otimes \Lambda /\left(p^{s}, f\right)$, which is obtained by propagating $\mathcal{F}_{\text {can }}^{\Lambda}$ (in the sense of Definition 2.1 ), will also be denoted by $\mathcal{F}_{\text {can }}^{\Lambda}$.

Let $T_{s, m}:=T \otimes \Lambda /\left(p^{s}, \mathbf{X}^{m}\right)$, where $\mathbf{X}$ is as in Section 2.3.2.

REMARK 2.35. By the definition of $\mathcal{F}_{\mathbb{L}}$, the local conditions on $T_{s, m}$ at primes $\lambda \nmid p$ propagated from $\mathcal{F}_{\mathbb{L}}$ coincide with the local conditions propagated from $\mathcal{F}_{\Lambda}$, and thanks to [B3, Corollaries 2.8, 2.9], they also coincide with the local conditions determined by $\mathcal{F}_{\text {can }}^{\Lambda}$, since $\left(\mathbb{H} . \mathbf{T}_{/ k}\right)$ holds true. Indeed, it is proved in [B3] that all these local conditions coincide with

$$
H_{\mathrm{unr}}^{1}\left(k_{\lambda}, T_{s, m}\right):=\operatorname{ker}\left\{H^{1}\left(k_{\lambda}, T_{s, m}\right) \longrightarrow H^{1}\left(k_{\lambda}^{\mathrm{unr}}, T_{s, m}\right)\right\},
$$

as long as hypothesis $\left(\mathbb{H}\right.$. $\left.\mathbf{T}_{/ k}\right)$ holds true. We note further that $\mathcal{F}_{\mathbb{L}}$ propagates to the Selmer structure $\mathcal{F}_{\mathcal{L}}$ on $T=T \otimes \Lambda /(\mathbf{X})$.

Definition 2.36 (compare [MR1, Definition 3.1.6]). We define the module of $\mathbb{L}$-restricted $\Lambda$-adic Kolyvagin systems to be

$$
\overline{\mathbf{K S}}\left(T \otimes \Lambda, \mathcal{F}_{\mathbb{L}}, \mathcal{P}\right):=\lim _{s, m} \underset{j}{\lim } \mathbf{K S}\left(T_{s, m}, \mathcal{F}_{\mathbb{L}}, \mathcal{P}_{j}\right)
$$


where $\mathbf{K S}\left(T_{s, m}, \mathcal{F}_{\mathbb{L}}, \mathcal{P}_{j}\right)$ is the module of Kolyvagin systems for the Selmer structure $\mathcal{F}_{\mathbb{L}}$ on the representation $T_{s, m}$.

Theorem 2.37. Suppose that $\left(\mathbb{H} . \mathbf{T}_{/ k}\right)$ and $(\mathbb{H} . \mathbf{s E Z} / k)$ hold true. Then the $\Lambda$-module $\overline{\mathbf{K S}}\left(T \otimes \Lambda, \mathcal{F}_{\mathbb{L}}, \mathcal{P}\right)$ is free of rank 1 , and the canonical map

$$
\overline{\mathbf{K S}}\left(T \otimes \Lambda, \mathcal{F}_{\mathbb{L}}, \mathcal{P}\right) \longrightarrow \overline{\mathbf{K S}}\left(T, \mathcal{F}_{\mathcal{L}}, \mathcal{P}\right)
$$

is surjective.

Note that, thanks to assumption $(\mathrm{A} 1)$, both $\left(\mathbb{H} . \mathbf{T}_{/ k}\right)$ and $(\mathbb{H} . \mathbf{s E Z} / k)$ are true for the particular Galois representation $T$ we are interested in. Any generator of the cyclic $\Lambda$-module $\overline{\mathbf{K S}}\left(T \otimes \Lambda, \mathcal{F}_{\mathbb{L}}, \mathcal{P}\right)$ will be called a primitive $\Lambda$-adic Kolyvagin system.

Proof. The proof of this theorem is very similar to the proof of [B2, Theorem 2.19], to which we refer the reader for details. We only remark here that the proof follows from an appropriate variant of [B3, Theorem 3.23], which applies (with the base field $\mathbb{Q}$ replaced by $k$, and the Selmer structure $\mathcal{F}_{\text {can }}$ replaced by $\mathcal{F}_{\mathbb{L}}$ ) thanks to Proposition 2.26 and the truth of hypotheses (H.1)-(H.4), (HI.T/k), and $(\mathbb{H} . \mathbf{s E Z} / k)$.

In Section 4.2 below, we explain how to obtain these Kolyvagin systems out of the Stickelberger elements, assuming a weak version of Brumer's conjecture. Note, however, that the existence of $\Lambda$-adic Kolyvagin systems does not rely on Brumer's conjecture.

We record here the main application of a $\Lambda$-adic Kolyvagin system

$$
\kappa=\left\{\left\{\kappa_{\tau}(s, m)\right\}_{\tau \in \mathcal{N}_{s+m}}\right\}_{s, m} .
$$

For an explanation of our notation, see [MR1, Section 3]. Here we only note that $\kappa_{\tau}(s, m) \in H^{1}\left(k, T_{s, m}\right)$, and by definition, there is a well-defined element

$$
\kappa_{1}=\left\{\kappa_{1}(s, m)\right\}_{s, m} \in \lim _{s, m} H_{\mathcal{F}_{\mathbb{L}}}^{1}\left(k, T_{s, m}\right)=H_{\mathcal{F}_{\mathbb{L}}}^{1}(k, T \otimes \Lambda) .
$$

For notational simplicity, we write $\mathbb{T}=T \otimes \Lambda$. Recall that $\operatorname{char}(\mathbb{A})$ denotes the characteristic ideal of a finitely generated $\Lambda$-module $\mathbb{A}$, with the convention that $\operatorname{char}(\mathbb{A})=0$ unless $\mathbb{A}$ is $\Lambda$-torsion.

THEOREM 2.38. Under assumptions (A1) and (A2),

$$
\operatorname{char}\left(H_{\mathcal{F}_{\mathbb{L}}^{*}}^{1}\left(k, \mathbb{T}^{*}\right)^{\vee}\right) \mid \operatorname{char}\left(H_{\mathcal{F}_{\mathbb{L}}}^{1}(k, \mathbb{T}) / \Lambda \cdot \kappa_{1}\right)
$$


Proof. This is [MR1, Theorem 5.3.10(iii)] applied in our setting. We remark that all the hypotheses of [MR1, Theorem 5.3.10(iii)] hold thanks to (A1) and (A2) (as we have already demonstrated above).

\section{§3. Euler systems from Stickelberger elements}

We begin by recalling the definition of Stickelberger elements. We first set our notation. Assume that $k, \chi, \mathfrak{f}=\mathfrak{f}_{\chi}$ and $L$ are as above. For a (square-free) cycle $\tau=\mathfrak{q}_{1} \cdots \mathfrak{q}_{m}$ of the number field $k$, let $k(\tau)$ be the compositum

$$
k(\tau)=k\left(\mathfrak{q}_{1}\right) \cdots k\left(\mathfrak{q}_{m}\right)
$$

where $k(\mathfrak{q})$ denotes the maximal $p$-extension inside the ray class field of $k$ modulo the prime ideal $\mathfrak{q}$. For any field $K$, define $K(\tau)$ as the composite of $k(\tau)$ and $K$. Let

$$
\begin{aligned}
& \mathcal{K}=\left\{L_{n}(\tau): \tau \in \mathcal{N} ; n \geq 0\right\}, \\
& \mathcal{K}_{0}=\left\{k_{n}(\tau): \tau \in \mathcal{N} ; n \geq 0\right\}
\end{aligned}
$$

be two collections of abelian extensions of $k$. Note that any field $L_{n}(\tau) \in \mathcal{K}$ is CM and abelian over the totally real field $k$. Let $S$ be the set of places of $k$, consisting of all places above $p$, all places dividing $\mathfrak{f}$, and all infinite places. For any $K \in \mathcal{K}$, write $S_{K}$ for the set of all places of the field $K$ lying above the places in $S$. When there is no confusion, we will simply write $S$ for $S_{K}$.

For any $K \in \mathcal{K}$, the partial zeta function for $\sigma \in \operatorname{Gal}(K / k)$ is defined as usual by

$$
\zeta_{S}(s, \sigma):=\sum_{\substack{(\mathfrak{a}, K / k)=\sigma \\ \mathfrak{a} \text { is prime to } S}} \mathbf{N a}^{-s}
$$

for $\operatorname{Re}(s)>1$. Here $\mathbf{N a}$ is the absolute norm of the ideal $\mathfrak{a} \in k$, and $(\mathfrak{a}, K / k)$ is the Artin symbol. The partial zeta functions admit a meromorphic continuation to the whole complex plane and are holomorphic everywhere except at $s=1$. We may therefore set

$$
\theta_{K}=\theta_{K, S}:=\sum_{\sigma \in \operatorname{Gal}(K / k)} \zeta_{S}(0, \sigma) \sigma^{-1} \in \mathbb{C}[\operatorname{Gal}(K / k)]
$$

Thanks to $[\mathrm{S}], \theta_{K}$ is an element of $\mathbb{Q}[\mathrm{Gal}(K / k)]$. Further, we know for the $\chi$ part $\theta_{K}^{\chi}$ of $\theta_{K}$, thanks to $[\mathrm{DR}]$, that $\theta_{K}^{\chi} \in \mathbb{Z}_{p}[\operatorname{Gal}(K / k)]^{\chi}$, since we assumed that $\chi \neq \omega$. 
Lemma 3.1. For any $L_{n}(\tau)=K \subset K^{\prime}=L_{n^{\prime}}\left(\tau^{\prime}\right)$ inside $\mathcal{K}$,

$$
\left.\theta_{K^{\prime}}\right|_{K}=\prod_{\mathfrak{q} \mid \tau^{\prime}, \mathfrak{q} \nmid \tau}\left(1-\operatorname{Fr}_{\mathfrak{q}}^{-1}\right) \theta_{K}
$$

Proof. This follows from [T, proposition IV.1.8].

As before, let $A_{K}$ denote the $p$-part of the ideal class group of $K \in \mathcal{K}$, and let $A_{K}^{\chi}$ denote its $\chi$-isotypic part. Until the end of this section, we suppose that the $\chi$-part of the Brumer's conjecture (Assumption 1.1) holds true.

Remark 3.2. Greither [Gr, Corollary 2.7] and Kurihara [Ku, Corollary 2.4] have deduced Assumption 1.1 from Iwasawa's main conjecture in this setting (which holds thanks to [W2]) and the vanishing of the Iwasawa $\mu$-invariant for $K$. However, we do not wish to assume the truth of the main conjecture; in fact, we rather assume in this paper Assumption 1.1 and deduce the main conjecture itself.

Having referred the reader to $[\mathrm{Ku}]$, we caution the reader about one minor point: If a prime $\wp \subset k$ above $p$ is unramified in $K / k$, then Kurihara's Stickelberger element $\tilde{\theta}_{K}^{\chi}$ differs from our $\theta_{K}^{\chi}$ by a factor of $\left(1-\operatorname{Fr}_{\wp}\right)^{\chi}$, where $\operatorname{Fr}_{\wp}$ is the Frobenius at $\wp$ for the unramified extension $K / k$. If (A1) holds, it follows that $\left(1-\mathrm{Fr}_{\wp}\right)^{\chi}$ is a unit inside $\mathbb{Z}_{p}[\operatorname{Gal}(K / k)]^{\chi}$. Therefore, the statement of Assumption 1.1 is still equivalent to the statement that $\tilde{\theta}_{K}^{\chi} \cdot A_{K}^{\chi}=0$, which is the assertion deduced from the main conjecture in $[\mathrm{Ku}]$.

Suppose that $F$ is any finite abelian extension of $k$ and that $K=F L$. Then by the inflation-restriction sequence and class field theory, one has

$$
H^{1}\left(F, \mathbb{Z}_{p}(\chi)\right) \cong H^{1}\left(K, \mathbb{Z}_{p}\right)^{\chi^{-1}}=\operatorname{Hom}\left(\mathbb{A}_{K}^{\times} / K^{\times}, \mathbb{Z}_{p}\right)^{\chi^{-1}}
$$

where $\mathbb{A}_{K}^{\times}$denotes the idèles of $K$. Since any continuous homomorphism of $\mathbb{A}_{K}^{\times}$into $\mathbb{Z}_{p}$ should vanish on

$$
B_{K}:=\prod_{w \mid \infty} K_{w}^{\times} \times \prod_{w \mid p}\{1\} \times \prod_{w \nmid p \infty} \mathcal{O}_{K_{w}}^{\times} \subset \mathbb{A}_{K}^{\times},
$$

(3.1) gives

$$
\begin{aligned}
H^{1}\left(F, \mathbb{Z}_{p}(\chi)\right) & \cong \operatorname{Hom}\left(\mathbb{A}_{K}^{\times} / K^{\times} B_{K}, \mathbb{Z}_{p}\right)^{\chi^{-1}} \\
& =\operatorname{Hom}\left(\left(\mathbb{A}_{K}^{\times} / K^{\times} B_{K}\right)^{\chi}, \mathbb{Z}_{p}\right) .
\end{aligned}
$$


Further, there is an exact sequence

$$
0 \longrightarrow U_{K} \overline{\mathcal{O}_{K}^{\times}} \longrightarrow \mathbb{A}_{K}^{\times} / K^{\times} B_{K} \longrightarrow A_{K} \longrightarrow 0
$$

which is induced from the map that sends an idèle to the corresponding ideal class. Here $\overline{\mathcal{O}_{K}^{\times}}$is the closure of the global units $\mathcal{O}_{K}^{\times}$inside the local units $U_{K} \subset K \otimes \mathbb{Q}_{p}$. Since taking $\chi$-parts is exact (as the order of $\chi$ is prime to $p$ ), we obtain an exact sequence

$$
0 \longrightarrow U_{K}^{\chi} /\left(\overline{\mathcal{O}_{K}^{\times}}\right)^{\chi} \longrightarrow\left(\mathbb{A}_{K}^{\times} / K^{\times} B_{K}\right)^{\chi} \longrightarrow A_{K}^{\chi} \longrightarrow 0
$$

Thus, by Assumption 1.1, multiplication by $\theta_{K}^{\chi}$ gives a map

$$
\left(\mathbb{A}_{K}^{\times} / K^{\times} B_{K}\right)^{\chi} \stackrel{\theta_{K}^{\chi}}{\longrightarrow} U_{K}^{\chi} /\left(\overline{\mathcal{O}_{K}^{\times}}\right)^{\chi}
$$

Since we assumed that $\chi$ is totally odd, $\left(\overline{\mathcal{O}_{K}^{\times}}\right)^{\chi}$ is finite (see the final paragraph of the proof of Proposition 2.13), and we therefore have an induced map

$$
\left(\mathbb{A}_{K}^{\times} / K^{\times} B_{K}\right)^{\chi} \stackrel{\theta_{K}^{\chi}}{\longrightarrow} U_{K}^{\chi} /\left(U_{K}^{\chi}\right)_{\text {tors }}
$$

Suppose that we are given a collection of homomorphisms $\boldsymbol{\lambda}=\left\{\lambda_{n}^{\tau}\right\}$ with $\lambda_{n}^{\tau} \in \operatorname{Hom}\left(U_{L_{n}(\tau)}^{\chi}, \mathbb{Z}_{p}\right)$ which satisfies the following properties.

(1) For all $L_{n}(\tau), L_{n^{\prime}}(\tau \mathfrak{q}) \in \mathcal{K}$, the following diagram commutes:

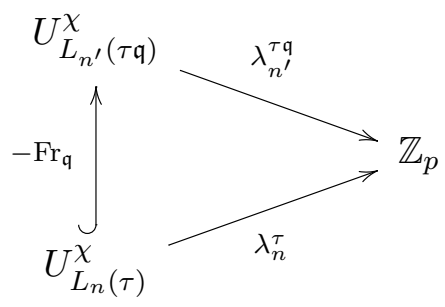

(2) For $n^{\prime} \geq n$, we have $\left.\lambda_{n^{\prime}}^{\tau}\right|_{U_{L_{n}(\tau)}^{\chi}}=\lambda_{n}^{\tau}$.

Define

$$
\tilde{c}_{k_{n}(\tau)} \in \operatorname{Hom}\left(\left(\mathbb{A}_{L_{n}(\tau)}^{\times} /\left(L_{n}(\tau)\right)^{\times} B_{L_{n}(\tau)}\right)^{\chi}, \mathbb{Z}_{p}\right)
$$


(which we view also as an element of $H^{1}\left(k_{n}(\tau), T\right)$ via identification (3.2) above) as the composition*

$$
\tilde{c}_{k_{n}(\tau)}:\left(\mathbb{A}_{L_{n}(\tau)}^{\times} /\left(L_{n}(\tau)\right)^{\times} B_{L_{n}(\tau)}\right)^{\chi \stackrel{\theta_{L_{n}(\tau)}^{\chi}}{\longrightarrow}} U_{L_{n}(\tau)}^{\chi} /\left(U_{L_{n}(\tau)}\right)_{\mathrm{tors}}^{\chi} \stackrel{\lambda_{n}^{\tau}}{\longrightarrow} \mathbb{Z}_{p}
$$

Set $\tilde{\mathbf{c}}=\left\{\tilde{c}_{k_{n}(\tau)}\right\}$.

THEOREM 3.3. Fix a collection of homomorphisms $\boldsymbol{\lambda}=\left\{\lambda_{n}^{\tau}\right\}$ as above (if it exists). Then there is an Euler system $\mathbf{c}=\left\{c_{k_{n}(\tau)}\right\}$ (which depends on the choice of $\boldsymbol{\lambda}$ ) for the Galois representation $T$ (in the sense of [R3, Definition II.1.1, Remark II.1.4]) such that $c_{k_{n}}=\tilde{c}_{k_{n}}$ for all $n$.

In Section 4.1 below, we construct a collection $\boldsymbol{\lambda}$ which satisfies the desired properties and hence conclude with the existence of an Euler system for $T$, assuming the truth of Assumption 1.1. When $k=\mathbb{Q}$, this Euler system has been given by Rubin [R3, Section 3.4].

Proof. Since the proof of this theorem very closely follows the proof of [R3, Proposition III.3.4], we give only a sketch. All the references in this proof are to [R3]. First, one checks (mimicking the proof of [R3, Proposition III.3.4]) that the collection $\tilde{\mathbf{c}}$ (which should be compared with the collection $\tilde{\mathbf{c}}^{\prime}$ of Rubin) satisfies a distribution relation with wrong Euler factors. This could be remedied, as in the paragraph following [R3, Remark III.4.4], using [R3, Lemma IX.6.1] to obtain a new collection c (which corresponds to what Rubin calls $\tilde{\mathbf{c}}$ ), as desired.

We close this section with a final remark which we will refer to in what follows.

REMARK 3.4. The argument of Remark 2.16 shows that, under hypothesis (A1),

$$
H^{1}\left(k_{n}(\tau)_{p}, T\right) \cong \operatorname{Hom}\left(U_{L_{n}(\tau)}^{\chi}, \mathbb{Z}_{p}\right) .
$$

REMARK 3.5. In this remark we discuss the main differences between the cases when the base field $k$ is a general totally real field (i.e., the case we study in this article) and the particular case $k=\mathbb{Q}$ (i.e., the case Rubin studies in [R3, Section III.4]).

${ }^{*}$ We remark that any homomorphism $\lambda \in \operatorname{Hom}\left(U_{L_{n}(\tau)}^{\chi}, \mathbb{Z}_{p}\right)$ necessarily factors through the quotient $U_{L_{n}(\tau)}^{\chi} /\left(U_{L_{n}(\tau)}\right)_{\text {tors }}^{\chi}$; this is how we make sense of the rightmost map in (3.4). 
(i) The first difference regards the core Selmer ranks. The core rank $\mathcal{X}\left(T, \mathcal{F}_{\text {can }}\right)$ of the canonical Selmer structure (see Example 2.6 above) of the $G_{k}$-representation $T$ is $[k: \mathbb{Q}]=r$. Rubin treats the case $r=1$ (i.e., the case $k=\mathbb{Q})$. In this paper, we study the case $r>1$, adapting the work of Mazur and Rubin [MR1] to the general case when $\mathcal{X}\left(T, \mathcal{F}_{\text {can }}\right)>$ 1 via what we call $\mathcal{L}$-restricted Euler systems. Although Kurihara $[\mathrm{Ku}]$ successfully applies the classical Euler system argument to Stickelberger elements to prove Theorems $\mathrm{A}$ and $\mathrm{B}$ above, our approach via $\mathcal{L}$ restricted Euler systems yields in addition a comparison between the Stickelberger elements and Rubin-Stark elements (see Theorem 5.16 below). Furthermore, our approach here fits well into the framework developed in [MR1] which was later enhanced by the author in [B1], [B2], and [B4].

(ii) The second difference is the manner in which the collection $\lambda=\left\{\lambda_{n}^{\tau}\right\}$ of homomorphisms is chosen. Rubin [R3, Appendix D] constructs these homomorphisms explicitly when $k=\mathbb{Q}$. This construction is not available when $k \neq \mathbb{Q}$; that is why we prove "abstractly" in Section 4.1 that a collection $\boldsymbol{\lambda}$ exists with the desired properties.

\section{$\S 4$. Euler systems to Kolyvagin systems map}

We first recall what Mazur and Rubin call the Euler system to Kolyvagin system map. Suppose that $T, \mathcal{K}$, and $\mathcal{P}$ are as above. Let $\mathbf{E S}(T)=$ $\operatorname{ES}(T, \mathcal{K})$ denote the collection of Euler systems for $(T, \mathcal{K})$ in the sense of [R3, Section 3]. Recall also the generalized module of Kolyvagin systems $\overline{\mathbf{K S}}(T, \mathcal{F}, \mathcal{P})$ and $\overline{\mathbf{K S}}(T \otimes \Lambda, \mathcal{F}, \mathcal{P})$ for various choices of Selmer structures $\mathcal{F}$.

Theorem 4.1 (Mazur and Rubin). There are canonical maps

- $\mathbf{E S}(T) \longrightarrow \overline{\mathbf{K S}}\left(T, \mathcal{F}_{\text {can }}, \mathcal{P}\right)$,

- $\mathbf{E S}(T) \longrightarrow \overline{\mathbf{K S}}\left(T \otimes \Lambda, \mathcal{F}_{\Lambda}, \mathcal{P}\right)$

with the following properties:

(1) if $\mathbf{c} \in \mathbf{E S}(T)$ maps to $\boldsymbol{\kappa} \in \overline{\mathbf{K S}}\left(T, \mathcal{F}_{\text {can }}, \mathcal{P}\right)$, then $\kappa_{1}=c_{k}$;

(2) if $\mathbf{c} \in \mathbf{E S}(T)$ maps to $\boldsymbol{\kappa} \in \overline{\mathbf{K S}}\left(T \otimes \Lambda, \mathcal{F}_{\Lambda}, \mathcal{P}\right)$, then

$$
\kappa_{1}=\left\{c_{k_{n}}\right\} \in{\underset{n}{n}}_{\lim } H^{1}\left(k_{n}, T\right)=H^{1}(k, T \otimes \Lambda) .
$$

Proof. Let $\rho_{\text {cyc }}: G_{k} \rightarrow \mathbb{Z}_{p}^{\times}$be the cyclotomic character (giving the action of $G_{k}$ on $\boldsymbol{\mu}_{p^{\infty}}$ ), and set

$$
\rho=\omega^{-1} \rho_{\text {cyc }}: \Gamma \longrightarrow 1+p \mathbb{Z}_{p}
$$


Let $\psi=\omega \chi^{-1}$ be as in the introduction, and set

$$
T^{\prime}=\mathbb{Z}_{p}(1) \otimes \psi^{-1}=T \otimes \rho, \quad \text { and } \quad \mathbb{T}^{\prime}=T^{\prime} \otimes \Lambda .
$$

Note that we have an isomorphism of $G_{k}$-modules

$$
\mathbb{T}^{\prime}=T^{\prime} \otimes \Lambda \stackrel{\otimes \rho^{-1}}{\longrightarrow} T \otimes \Lambda=\mathbb{T}
$$

as $\rho$ is a character of $\Gamma$. We then have the following diagram:

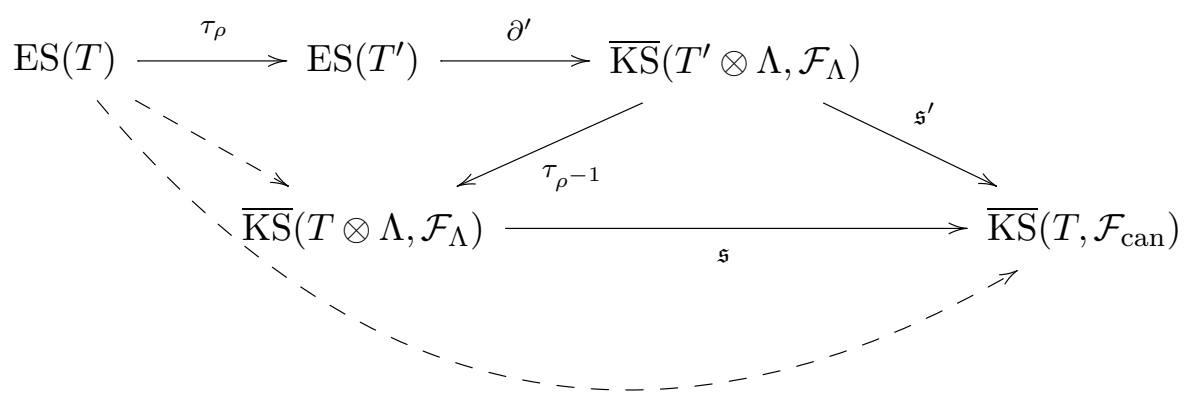

The two dashed arrows are the maps claimed to exist in the statement of the theorem, and they are given as the composition of relevant maps in the diagram. We now explain how the other arrows are obtained. The map $\tau_{\rho}$ is obtained by applying a formal twisting argument (see [R3, Section 6]). The map $\tau_{\rho^{-1}}$ is induced from isomorphism (4.1), and $\mathfrak{s}$ is induced from the specialization $T \otimes \Lambda \rightarrow T$ whose kernel is the augmentation ideal of $\Lambda$. Similarly, $\mathfrak{s}^{\prime}$ is induced from the specialization $T^{\prime} \otimes \Lambda \rightarrow T$ which makes the triangle

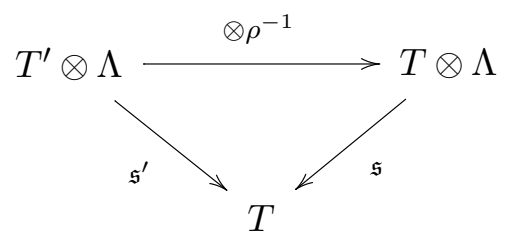

(as well as the triangle in the diagram above) commutative. The map $\partial^{\prime}$ is the Euler systems to Kolyvagin systems map of Mazur and Rubin [R3, Theorem 5.3.3], which is obtained as follows. Starting with an Euler system $\boldsymbol{c}^{\prime} \in \operatorname{ES}\left(T^{\prime}\right)$ for $T^{\prime}$, Kolyvagin's construction (see [R3, Section 4]) yields a weak Kolyvagin system (in the sense of [MR1, Definition 3.1.8]),

$$
\kappa^{\mathrm{w}}=\left\{\kappa_{\eta}^{\mathrm{w}}\right\}_{\eta \in \mathcal{N}}
$$


The classes $\kappa_{\eta}^{\mathrm{w}}$ do not necessarily satisfy the transverse local condition at a prime $\lambda \mid \eta$. One may, however, first calculate the finite projections of these classes (slightly modifying (by replacing $\mathbb{Q}(\ell)$ by $k(\lambda)$ and $\mathbb{Q}(n)$ by $k(\eta)$ where necessary) as in [MR1, Theorem A.4]; see particularly Lemma A.6 and Proposition A.8 for the key steps). Note that we pass to an auxiliary twist $T^{\prime}$ to ensure that $\operatorname{Fr}_{\lambda}^{p^{m}}-1$ acts injectively on $T^{\prime}$ for every $\lambda \in \mathcal{P}$ and for every $m \in \mathbb{Z}^{+}$, which is needed for the arguments of Mazur and Rubin to be performed. Finally, one may modify $\boldsymbol{\kappa}^{\mathrm{w}}$, as $[\mathrm{MR} 1,(33)]$ does to kill its finite projections and thus obtain a Kolyvagin system, as desired.

REMARK 4.2. Mazur and Rubin's definition of the generalized module of $\Lambda$-adic Kolyvagin systems $\overline{\mathbf{K S}}\left(T \otimes \Lambda, \mathcal{F}_{\Lambda}, \mathcal{P}\right)$ slightly differs from our definition of this module (Definition 2.36). It is not hard to see that these two definitions give rise to isomorphic modules (see also Remark 4.13 below).

We would like to apply this map to the Euler systems that we constructed* in Section 3. Note, however, that Theorem 4.1 will give rise to Kolyvagin systems only for the coarser Selmer structures $\mathcal{F}_{\Lambda}$ and $\mathcal{F}_{\text {can }}$ (rather than the finer Selmer structures $\mathcal{F}_{\mathbb{L}}$ and $\mathcal{F}_{\mathcal{L}}$ ). To be able to obtain Kolyvagin systems for the modified Selmer structures $\mathcal{F}_{\mathbb{L}}$ and $\mathcal{F}_{\mathcal{L}}$, we need to analyze the structure of the semilocal cohomology groups for $T \otimes \Lambda$ and $T$ over various ray class fields of $k$. This is performed in Section 4.1. We then apply the results of Section 4.1 to construct the desired Kolyvagin systems for the modified Selmer structures in Section 4.2.

REMARK 4.3. In effect, one needs only a weak Kolyvagin system (in the sense of [MR1, Definition 3.1.8]) for the main application of the Euler/ Kolyvagin system machinery, that is, for bounding the dual Selmer group. Weak Kolyvagin systems are essentially the derivative classes of Kolyvagin (see [R3, Section IV]) which are obtained by directly applying the derivative operators, without the need of the alterations performed in [MR1, Appendix A].

\subsection{A good choice of homomorphisms}

Recall that $k_{\infty}$ is the cyclotomic $\mathbb{Z}_{p}$-extension of $k$ and that $\Gamma=\operatorname{Gal}\left(k_{\infty} / k\right)$. Let $k_{n}$ denote the unique subextension of $k_{\infty} / k$ with $\left[k_{n}: k\right]=p^{n}$, and set $\Gamma_{n}:=\operatorname{Gal}\left(k_{n} / k\right)$. Recall also that $\Delta_{\tau}:=\operatorname{Gal}(k(\tau) / k)$.

\footnotetext{
${ }^{*}$ Modulo the existence of a family of homomorphisms $\boldsymbol{\lambda}$.
} 
Lemma 4.4. For every $n \in \mathbb{Z}_{\geq 0}$ and $\tau \in \mathcal{N}(\mathcal{P})$, the corestriction maps on the semilocal cohomology,

(i) $H^{1}\left(k_{n}(\tau)_{p}, T\right) \longrightarrow H^{1}\left(k(\tau)_{p}, T\right)$,

(ii) $H^{1}\left(k(\tau)_{p}, T\right) \longrightarrow H^{1}\left(k_{p}, T\right)$,

(iii) $H^{1}\left(k_{n}(\tau)_{p}, T\right) \longrightarrow H^{1}\left(k_{p}, T\right)$,

are surjective.

Proof. The cokernel of the map

$$
H^{1}(k(\tau), T \otimes \Lambda)={\underset{\lim }{n}}^{\operatorname{li}} H^{1}\left(k_{n}(\tau)_{p}, T\right) \longrightarrow H^{1}\left(k(\tau)_{p}, T\right)
$$

is given by $H^{2}\left(k(\tau)_{p}, T \otimes \Lambda\right)[\gamma-1]$, where $\gamma$ is any topological generator of $\Gamma=\operatorname{Gal}\left(k_{\infty} / k\right)$. Since it is known that $H^{2}\left(k(\tau)_{p}, T \otimes \Lambda\right)$ is a finitely generated $\mathbb{Z}_{p}$-module (see $[\mathrm{P} 1]$ ), it follows that

$$
H^{2}\left(k(\tau)_{p}, T \otimes \Lambda\right)[\gamma-1]=0 \Longleftrightarrow H^{2}\left(k(\tau)_{p}, T \otimes \Lambda\right) /(\gamma-1)=0 .
$$

Since the cohomological dimension of the absolute Galois group of any local field is 2 ,

$$
H^{2}\left(k(\tau)_{p}, T \otimes \Lambda\right) /(\gamma-1) \cong H^{2}\left(k(\tau)_{p}, T \otimes \Lambda /(\gamma-1)\right)=H^{2}\left(k(\tau)_{p}, T\right) .
$$

It therefore suffices to check that

$$
H^{2}\left(k(\tau)_{p}, T\right):=\bigoplus_{v \mid p} H^{2}\left(k(\tau)_{v}, T\right)=0,
$$

which, via local duality, is equivalent to checking that $\left(T^{*}\right)^{G_{k(\tau)} v}=0$ for each $v \mid p$.

Write $\mathcal{D}_{v}$ for the decomposition group at $v \mid p$ inside $\operatorname{Gal}(k(\tau) / k):=\Delta_{\tau}$. We may identify $\mathcal{D}_{v} \subset \Delta_{\tau}$ by the local Galois group $\operatorname{Gal}\left(k(\tau)_{v} / k_{\wp}\right)$, where $\wp \subset k$ is the prime below $v$. Since $\Delta_{\tau}$ is generated by inertia groups at the primes dividing $\tau$, all of these act trivially on $T^{*}$ (by the choice of $\tau$ ). Hence, it follows that

$$
\left(T^{*}\right)^{G_{k(\tau)}}=\left(T^{*}\right)^{G_{k_{\wp}}} .
$$

Note that $T^{*}=\boldsymbol{\mu}_{p^{\infty}} \otimes \chi^{-1}$, so it follows at once that $\left(T^{*}\right)^{G_{k_{\wp}}}=0$, and thus (i) is proved.

Set $T_{\tau}:=\operatorname{Ind}_{k(\tau)}^{k} T$. The semilocal version of Shapiro's lemma (which is explained in [R3, Section A.5]) shows that

$$
H^{1}\left(k(\tau)_{p}, T\right) \cong H^{1}\left(k_{p}, T_{\tau}\right) .
$$


The corestriction map

$$
\mathbf{N}_{\tau}: H^{1}\left(k_{p}, T_{\tau}\right) \cong H^{1}\left(k(\tau)_{p}, T\right) \longrightarrow H^{1}\left(k_{p}, T\right)
$$

is simply induced from the augmentation sequence

$$
0 \longrightarrow \mathcal{A}_{\tau} \cdot T_{\tau} \longrightarrow T_{\tau} \longrightarrow T \longrightarrow 0
$$

where $\mathcal{A}_{\tau}$ is the augmentation ideal of the local ring $\mathbb{Z}_{p}\left[\Delta_{\tau}\right]$. The argument above shows that the cokernel of $\mathbf{N}_{\tau}$ is dual to $H^{0}\left(k_{p},\left(\mathcal{A}_{\tau} \cdot T_{\tau}\right)^{*}\right)$. Furthermore,

$$
\left(\mathcal{A}_{\tau} \cdot T_{\tau}\right)^{*}:=\operatorname{Hom}\left(\mathcal{A}_{\tau} \cdot T_{\tau}, \boldsymbol{\mu}_{p^{\infty}}\right)=\operatorname{Hom}\left(\mathcal{A}_{\tau} \cdot T_{\tau}, \mathbb{Q}_{p} / \mathbb{Z}_{p}\right) \otimes \mathbb{Z}_{p}(1)
$$

and $\operatorname{Hom}\left(\mathcal{A}_{\tau} \cdot T_{\tau}, \mathbb{Q}_{p} / \mathbb{Z}_{p}\right)=\mathcal{A}_{\tau} \cdot \operatorname{Hom}\left(T_{\tau}, \mathbb{Q}_{p} / \mathbb{Z}_{p}\right)$; we thence see that

$$
H^{0}\left(k_{p},\left(\mathcal{A}_{\tau} \cdot T_{\tau}\right)^{*}\right) \hookrightarrow H^{0}\left(k_{p}, T_{\tau}^{*}\right) .
$$

It therefore suffices to show that $H^{0}\left(k_{p}, T_{\tau}^{*}\right)=0$. By local duality, this is equivalent to proving that $H^{2}\left(k_{p}, T_{\tau}\right)=0$, which, by the semilocal version of Shapiro's lemma, is equivalent to checking that $H^{2}\left(k(\tau)_{p}, T\right)=0$. This final statement is equivalent to the assertion that $H^{0}\left(k(\tau)_{p}, T^{*}\right)=0$ by local duality. This, however, has been already verified in the third paragraph of this proof. This completes the proof of (ii).

Assertion (iii) clearly follows from (i) and (ii).

Proposition 4.5. For every $\tau \in \mathcal{N}(\mathcal{P})$,

(i) the semilocal cohomology group $H^{1}\left(k(\tau)_{p}, T\right)$ is a free $\mathbb{Z}_{p}\left[\Delta_{\tau}\right]$-module of rank $r$,

(ii) for every $n \in \mathbb{Z}_{\geq 0}$, the $\mathbb{Z}_{p}\left[\Gamma_{n} \times \Delta_{\tau}\right]$-module $H^{1}\left(k_{n}(\tau)_{p}, T\right)$ is free of rank $r$.

Proof. We start with the remark that $H^{1}\left(k(\tau)_{p}, T\right)$ is a free $\mathbb{Z}_{p}$-module of rank $r \cdot\left|\Delta_{\tau}\right|$. Indeed, this may be proved by the argument of Lemma 2.15 (or, alternatively and more directly, following the argument of Remark 2.16). Further, we know thanks to Lemma 4.4 that the map

$$
H^{1}\left(k(\tau)_{p}, T\right) \longrightarrow H^{1}\left(k_{p}, T\right)
$$

(which could be thought of as the reduction modulo the augmentation ideal $\mathcal{A}_{\tau}$ ) is surjective. Nakayama's lemma and Lemma 2.15 therefore imply that 
$H^{1}\left(k(\tau)_{p}, T\right)$ is generated by (at most) $r$ elements over $\mathbb{Z}_{p}\left[\Delta_{\tau}\right]$. Let $\mathfrak{B}=$ $\left\{x_{1}, x_{2}, \ldots, x_{r}\right\}$ be any set of such generators. To prove (i), it suffices to check that the $x_{i}$ do not admit any $\mathbb{Z}_{p}\left[\Delta_{\tau}\right]$-linear relation. Assume the contrary, and suppose that there is a relation

$$
\sum_{i=1}^{r} \alpha_{i} x_{i}=0, \quad \alpha_{i} \in \mathbb{Z}_{p}\left[\Delta_{\tau}\right]
$$

Define

$$
S=\left\{\delta x_{j}: \delta \in \Delta_{\tau}, 1 \leq j \leq r\right\}
$$

and note that $S$ generates (as a $\mathbb{Z}_{p}$-module) $H^{1}\left(k(\tau)_{p}, T\right)$ by our assumption on $\mathfrak{B}$, and also that $|S|=r \cdot\left|\Delta_{\tau}\right|=\operatorname{rank}_{\mathbb{Z}_{p}}\left(H^{1}\left(k(\tau)_{p}, T\right)\right)$. Equation (4.2) can be rewritten as

$$
\sum_{\delta, j} a_{\delta, j} \cdot \delta x_{j}=0
$$

with $a_{\delta, j} \in \mathbb{Z}_{p}$. Since we already know that $H^{1}\left(k(\tau)_{p}, T\right)$ is $\mathbb{Z}_{p}$-torsion free, we may assume without loss of generality that $a_{\delta_{0}, j_{0}} \in \mathbb{Z}_{p}^{\times}$for some $\delta_{0}, j_{0}$. This in turn implies that

$$
\delta_{0} x_{j_{0}} \in \operatorname{span}_{\mathbb{Z}_{p}}\left(S-\left\{\delta_{0} x_{j_{0}}\right\}\right)
$$

hence, $H^{1}\left(k(\tau)_{p}, T\right)$ is generated by $S-\left\{\delta_{0} x_{j_{0}}\right\}$. This, however, is a contradiction since we already know that the $\mathbb{Z}_{p}$-rank of $H^{1}\left(k(\tau)_{p}, T\right)$ is $r \cdot\left|\Delta_{\tau}\right|=$ $|S|$; hence, it cannot be generated by $|S|-1$ elements over $\mathbb{Z}_{p}$. The proof of (i) now follows.

One proves (ii) in an identical fashion, now considering the augmentation map

$$
H^{1}\left(k_{n}(\tau)_{p}, T\right) \longrightarrow H^{1}\left(k(\tau)_{p}, T\right)
$$

which is surjective thanks to Lemma 4.4 .

Define the field $\mathfrak{F}$ as the compositum of the fields $k(\tau)$,

$$
\mathfrak{F}=\bigcup_{\tau \in \mathcal{N}(\mathcal{P})} k(\tau),
$$

as $\tau$ runs through the set $\mathcal{N}$. We set $\Delta:=\operatorname{Gal}(\mathfrak{F} / k)$. 
Corollary 4.6. The $\mathbb{Z}_{p}[[\Gamma \times \Delta]]$-module $\varliminf_{n, \tau} H^{1}\left(k_{n}(\tau)_{p}, T\right)$ is free of rank $r$, and the natural projection

$$
\lim _{n, \tau} H^{1}\left(k_{n}(\tau)_{p}, T\right) \longrightarrow H^{1}\left(k_{m}(\eta)_{p}, T\right)
$$

is surjective for every $m \in \mathbb{Z}_{\geq 0}$ and $\eta \in \mathcal{N}$.

Proof. This is immediate after Proposition 4.5.

Definition 4.7. Fix a $\mathbb{Z}_{p}[[\Gamma \times \boldsymbol{\Delta}]]$-rank 1 direct summand $\mathcal{L}$ of

$$
\lim _{n, \tau} H^{1}\left(k_{n}(\tau)_{p}, T\right)
$$

Denote its image under the (surjective) map

$$
\lim _{n, \tau} H^{1}\left(k_{n}(\tau)_{p}, T\right) \longrightarrow H^{1}\left(k_{m}(\eta)_{p}, T\right)
$$

by $\mathcal{L}_{m}^{\eta}$. When $\eta=1$, we simply write $\mathcal{L}_{m}$ instead of $\mathcal{L}_{m}^{1}$, and when $m=0$, we write $\mathcal{L}$ for $\mathcal{L}_{0}$. Finally, let $\mathbb{L}$ denote the image of $\mathcal{L}$ under the projection

$$
\lim _{n, \tau} H^{1}\left(k_{n}(\tau)_{p}, T\right) \longrightarrow \lim _{n} H^{1}\left(\left(k_{n}\right)_{p}, T\right)=H^{1}\left(k_{p}, T \otimes \Lambda\right)
$$

We fix generators $\varphi, \varphi_{m}^{\eta}, \varphi_{m}, \varphi$, and $\Phi$ of $\mathcal{L}, \mathcal{L}_{m}^{\eta}, \mathcal{L}_{m}, \mathcal{L}$, and $\mathbb{L}$, respectively, such that

$$
\varphi \mapsto \varphi_{m}^{\eta} \mapsto \varphi_{m}, \quad \text { and } \quad \varphi \mapsto \Phi \mapsto \varphi
$$

under the projection maps we mentioned above.

As in Definition 4.7, we could start with a choice of $\mathcal{L}$, which in turn fixes $\mathbb{L}$ and $\mathcal{L}$. Alternatively, we could start with an arbitrary $\mathcal{L}$ (and $\mathbb{L}$ ) as we did in Section 2.3 and show (using linear algebra) that there is a rank 1 direct summand $\mathcal{L} \subset \lim _{n, \tau} H^{1}\left(k_{n}(\tau)_{p}, T\right)$ which projects down to $\mathcal{L}$ (and $\mathbb{L})$, as in Definition 4.7.

REMARK 4.8. In Definition 2.27 (resp., Definition 2.36) above, we give a definition of an $\mathcal{L}$-restricted Kolyvagin system (resp., $\mathbb{L}$-restricted $\Lambda$-adic Kolyvagin system). When $\mathcal{L}$ is above, so that the line $\mathcal{L}$ projects down to $\mathcal{L}$ (resp., down to $\mathbb{L}$ ), we also call these $\mathcal{L}$-restricted Kolyvagin systems.

By Remark 3.4, we may identify $\lim _{n, \tau} H^{1}\left(k_{n}(\tau)_{p}, T\right)$ by the module $\lim _{n, \tau} \operatorname{Hom}\left(U_{L_{n}(\tau)}^{\chi}, \mathbb{Z}_{p}\right)$, where we recall that $U_{L_{n}(\tau)}$ stands for the local units 
inside $L_{n}(\tau) \otimes \mathbb{Q}_{p}$. We define, for each $m \geq 0$ and $\eta \in \mathcal{N}$, a homomorphism $\lambda_{m}^{\eta} \in \operatorname{Hom}\left(U_{L_{m}(\eta)}^{\chi}, \mathbb{Z}_{p}\right)$ as the composite

$$
\lambda_{m}^{\eta}:=\varphi_{m}^{\eta} \circ \prod_{\mathfrak{q} \mid \eta}\left(-\operatorname{Fr}_{\mathfrak{q}}\right) .
$$

We further set $\beth_{m}^{\eta}$ for the (free of rank 1) $\mathbb{Z}_{p}\left[\Gamma_{m} \times \Delta_{\eta}\right]$-module generated by $\lambda_{m}^{\eta}$. Clearly,

$$
\beth_{m}^{\eta}=\prod_{\mathfrak{q} \mid \eta}\left(-\mathrm{Fr}_{\mathfrak{q}}^{-1}\right) \mathcal{L}_{m}^{\eta}=\mathcal{L}_{m}^{\eta}
$$

where the final equality is because $\mathcal{L}_{m}^{\eta}$ is a $\mathbb{Z}_{p}\left[\Gamma_{m} \times \Delta_{\eta}\right]$-stable submodule of

$$
H^{1}\left(k_{m}(\eta), T\right) \cong \operatorname{Hom}\left(U_{L_{m}(\eta)}^{\chi}, \mathbb{Z}_{p}\right) .
$$

When $\eta$ is fixed and $m$ varies, note that the collection $\left\{\lambda_{m}^{\eta}\right\}_{m}$ forms a projective system with respect to norm maps. ${ }^{*}$ When $\eta=1$, we write $\lambda_{m}$ (resp., $\beth_{m}$ ) instead of $\lambda_{m}^{\eta}$ (resp., $\beth_{m}^{\eta}$ ). Also, when $m=0$, we simply write $\lambda$ (resp., I) for $\lambda_{0}$ (resp., $\beth_{0}$ ).

We finally remark that $\lambda_{m}=\varphi_{m}$ for all $m$, by definition.

Proposition 4.9. For $\eta, \eta \mathfrak{q} \in \mathcal{N}$ and any $m^{\prime} \geq m$,

(i) $\left.\lambda_{m^{\prime}}^{\eta \mathfrak{q}}\right|_{U_{L_{m}(\eta)}^{\chi}}=\lambda_{m}^{\eta} \circ\left(-\mathrm{Fr}_{\mathfrak{q}}\right)$

(ii) $\left.\lambda_{m^{\prime}}^{\eta}\right|_{U_{L_{m}(\eta)}^{\chi}} ^{L_{m}(\eta)}=\lambda_{m}^{\eta}$.

Proof. This is evident, since by construction

$$
\begin{aligned}
\left.\lambda_{m^{\prime}}^{\eta \mathfrak{q}}\right|_{U_{L_{m}(\eta)}^{\chi}} & =\left.\varphi_{m^{\prime}}^{\eta \mathfrak{q}} \circ \prod_{\varpi \mid \eta}\left(-\mathrm{Fr}_{\varpi}\right)\left(-\mathrm{Fr}_{\mathfrak{q}}\right)\right|_{U_{L_{m}(\eta)}^{\chi}} \\
& =\left.\varphi_{m}^{\eta} \circ \prod_{\varpi \mid \eta}\left(-\mathrm{Fr}_{\varpi}\right)\left(-\mathrm{Fr}_{\mathfrak{q}}\right)\right|_{U_{L_{m}(\eta)}^{\chi}} \\
& =\left.\lambda_{m}^{\eta} \circ\left(-\mathrm{Fr}_{\mathfrak{q}}\right)\right|_{U_{L_{m}(\eta)}^{\chi}},
\end{aligned}
$$

where the second equality is because

$$
\left.\varphi_{m^{\prime}}^{\eta \mathfrak{q}}\right|_{U_{L_{m}(\eta)}^{\chi}}=\left.\varphi_{m}^{\eta}\right|_{U_{L_{m}(\eta)}^{\chi}}
$$

${ }^{*}$ Under identification (4.4), the norm maps on the cohomology are induced from the inclusions $U_{L_{m}(\eta)}^{\chi} \hookrightarrow U_{L_{m^{\prime}}(\eta)}^{\chi}, m^{\prime} \geq m$. 
by the norm coherence property of the collection $\left\{\varphi_{m}^{\eta}\right\}_{\eta, m}$. This completes (i), and (ii) is proved similarly.

Let $\mathbf{c}^{\mathrm{St}}=\left\{c_{k_{n}(\tau)}^{\mathrm{St}}\right\} \in \mathbf{E S}(T)$ be the Euler system constructed via Theorem 3.3 using the Stickelberger elements and the collection $\left\{\lambda_{m}^{\eta}\right\}$ we defined above. In the next section, we will use $\mathbf{c}^{\mathrm{St}}$ to construct a Kolyvagin system for the Selmer triple $\left(T, \mathcal{F}_{\mathcal{L}}, P\right)$ (resp., for the triple $\left.\left(T \otimes \Lambda, \mathcal{F}_{\mathbb{L}}, \mathcal{P}\right)\right)$.

Remark/DEFinition 4.10. Let $M$ be any $G_{k}$-representation which is free of finite rank as a $\mathbb{Z}_{p}$-module and which is unramified outside a finite set of places of $k$. Let $\mathcal{K}_{M}$ be a large abelian extension of $k$ defined as in [R3, Definition 1.1]. Suppose that $\mathfrak{S} \subset \lim _{K \subset \mathcal{K}_{M}} H^{1}\left(K_{p}, M\right)$ is any submodule. Let $\mathfrak{S}_{K} \subset H^{1}\left(K_{p}, M\right)$ denote the image of $\mathfrak{S}$ under the obvious projection map. We say that an Euler system

$$
\left\{c_{K}\right\}_{K \subset \mathcal{K}_{M}}=\mathbf{c} \in \mathbf{E S}\left(M, \mathcal{K}_{M}\right)=\mathbf{E S}(M)
$$

is $\mathfrak{S}$-restricted if $\operatorname{loc}_{p}\left(c_{K}\right) \in \mathfrak{S}_{K}$ for any finite extension $K \subset \mathcal{K}_{M}$ of $k$. The collection of $\mathfrak{S}$-restricted Euler systems for the pair $\left(M, \mathcal{K}_{M}\right)$ will be denoted by $\mathbf{E S}_{\mathfrak{S}}(M)=\mathbf{E S}_{\mathfrak{S}}\left(M, \mathcal{K}_{M}\right)$.

EXAMPLE 4.11.

(1) Let $\mathcal{L} \subset \lim _{n, \tau} H^{1}\left(k_{n}(\tau), T\right)$ be as in Definition 4.7. It is easy to see that the Euler system $\mathbf{c}^{\mathrm{St}}$ we construct above is an $\mathcal{L}$-restricted Euler system.

(2) Consider the even, nontrivial character $\omega \chi^{-1}:=\psi$ of $G_{k}$, and set $T^{\prime}:=$ $\mathbb{Z}_{p}(1) \otimes \psi^{-1}$. Only in this example, we let $\mathbb{L}$ denote a fixed $\mathbb{Z}_{p}[[\Gamma \times \Delta]]$ rank 1 direct summand of $\lim _{n, \tau} H^{1}\left(k_{n}(\tau), T^{\prime}\right)$. The author [B2, Section 3] has constructed an $\mathbb{L}$-restricted Euler system for the pair $\left(T^{\prime}, \mathcal{K}\right)$, starting from the conjectural Rubin-Stark elements.

Later in Section 5.3, we will construct another $\mathbb{L}$-restricted Euler system for $\left(T^{\prime}, \mathcal{K}\right)$, applying a formal twisting argument on the Euler system $\mathbf{c}^{\mathrm{St}}$. We will also compare this Euler system with the one coming from the Rubin-Stark elements, using the "rigidity" of the collection of $\Lambda$-adic Kolyvagin systems.

\subsection{Kolyvagin systems for modified Selmer groups (bis)}

Recall the sets $\mathcal{P}_{j} \subset \mathcal{P}$ and $\mathcal{N}_{j} \subset \mathcal{N}$. For notational simplicity, we write $\mathbb{T}:=T \otimes \Lambda$ from now on, and for a fixed topological generator $\gamma \in \Gamma$, we set $\gamma_{n}=\gamma^{p^{n}}$. Finally, let $\mathcal{M}$ be the maximal ideal of the ring $\Lambda$. 
Definition 4.12. For $\mathbb{F}=\mathcal{F}_{\Lambda}$ or $\mathcal{F}_{\mathbb{L}}$, we set

$$
\overline{\mathbf{K S}}^{\prime}(\mathbb{T}, \mathbb{F}, \mathcal{P}):=\lim _{m, n} \underset{j}{\lim } \mathbf{K S}\left(\mathbb{T} /\left(p^{m}, \gamma_{n}-1\right) \mathbb{T}, \mathbb{F}, \mathcal{P}_{j}\right)
$$

where $\mathbf{K S}\left(\mathbb{T} /\left(p^{m}, \gamma_{n}-1\right) \mathbb{T}, \mathbb{F}, \mathcal{P}_{j}\right)$ is the $\Lambda /\left(p^{m}, \gamma_{n}-1\right)$-module of Kolyvagin systems (in the sense of [MR1, Definition 3.1.3]) for the propagated Selmer structure $\mathbb{F}$ on the quotient $\mathbb{T} /\left(p^{m}, \gamma_{n}-1\right) \mathbb{T}$.

REMARK 4.13. We introduced the module $\overline{\mathbf{K S}}^{\prime}\left(\mathbb{T}, \mathcal{F}_{\Lambda}, \mathcal{P}\right)$ above because, after applying Kolyvagin's descent procedure (see [R3, Section IV]), one directly obtains elements of $\overline{\mathbf{K S}}^{\prime}\left(\mathbb{T}, \mathcal{F}_{\Lambda}, \mathcal{P}\right)$. On the other hand, it is not hard to see for $\mathbb{F}=\mathcal{F}_{\Lambda}$ or $\mathcal{F}_{\mathbb{L}}$ that the module $\overline{\mathbf{K S}}^{\prime}(\mathbb{T}, \mathbb{F}, \mathcal{P})$ defined above is naturally isomorphic to the module $\overline{\mathbf{K S}}(\mathbb{T}, \mathbb{F}, \mathcal{P})$ of Definition 2.36, using the fact that each of the collections $\left\{p^{m}, \gamma_{n}-1\right\}_{m, n}$ and $\left\{p^{m}, \mathbf{X}^{n}\right\}_{m, n}$ forms a base of neighborhoods at zero. Furthermore, using the fact that the collection $\left\{\mathcal{M}^{\alpha}\right\}_{\alpha \in \mathbb{Z}^{+}}$also forms a base of neighborhoods at zero, one may identify these two modules as Kolyvagin systems by the generalized module of Kolyvagin systems defined in [MR1, Definition 3.1.6]. By slight abuse, we will write $\overline{\mathbf{K S}}(\mathbb{T}, \mathbb{F}, \mathcal{P})$ for any of the three modules of Kolyvagin systems given by three different definitions (i.e., by Definitions 2.36 and 4.12 here, and [MR1, Definition 3.1.6]). For our purposes in this section, we will use Definition 4.12 to define this module.

Write

$$
\left\{\left\{\kappa_{\tau, m}^{\mathrm{St}}\right\}_{\tau \in \mathcal{N}_{m}}\right\}_{m}=\boldsymbol{\kappa}^{\mathrm{St}} \in \overline{\mathbf{K S}}\left(T, \mathcal{F}_{\text {can }}, \mathcal{P}\right)
$$

(resp.,

$$
\left.\left\{\left\{\kappa_{\tau}^{\mathrm{St}_{\infty}}(m, n)\right\}_{\tau \in \mathcal{N}_{m+n}}\right\}_{m, n}=\kappa^{\mathrm{St}} \in \overline{\mathbf{K S}}\left(\mathbb{T}, \mathcal{F}_{\Lambda}, \mathcal{P}\right)\right)
$$

for the Kolyvagin systems obtained via the descent procedure of [R3, Section 4] applied on the Euler system $\mathbf{c}^{\mathrm{St}}=\left\{c_{k_{n}(\tau)}^{\mathrm{St}}\right\}$. We know that

$$
\begin{aligned}
& \kappa_{1}^{\mathrm{St}} \stackrel{\text { def }}{=} \underset{m}{\lim } \kappa_{1, m}^{\mathrm{St}} \in \underset{m}{\lim } H^{1}\left(k, T / p^{m} T\right)=H^{1}(k, T) \\
& \| \\
& c_{k}^{\mathrm{St}} \stackrel{\text { def }}{=} \lambda \circ \theta_{L}^{\chi}=\varphi \circ \theta_{L}^{\chi} \in \operatorname{Hom}\left(\left(\mathbb{A}_{L}^{\times} / L^{\times}\right)^{\chi}, \mathbb{Z}_{p}\right)=H^{1}(k, T)
\end{aligned}
$$


and that

$$
\begin{gathered}
\kappa_{1}^{\mathrm{St}} \stackrel{\text { def }}{=} \underset{m, n}{\lim } \kappa_{1}(m, n) \in \underset{m, n}{\lim } H^{1}\left(k, \mathbb{T} /\left(p^{m}, \gamma_{n}-1\right) \mathbb{T}\right)=H^{1}(k, \mathbb{T}) \\
\| \\
\left\{c_{k_{n}}^{\mathrm{St}}\right\}_{n} \stackrel{\text { def }}{=}\left\{\lambda_{n} \circ \theta_{L_{n}}^{\chi}\right\}_{n}=\left\{\varphi_{n} \circ \theta_{L_{n}}^{\chi}\right\}_{n} \in \underset{n}{\lim } H^{1}\left(k_{n}, T\right)=H^{1}(k, \mathbb{T}) .
\end{gathered}
$$

REMARK 4.14. For every (rational) prime $\ell$, Shapiro's lemma shows that

$$
H^{1}\left(k(\tau), \mathbb{T} /\left(p^{m}, \gamma_{n}-1\right) \mathbb{T}\right) \cong H^{1}\left(k_{n}(\tau), T / p^{m} T\right)
$$

and

$$
H^{1}\left(k(\tau)_{\ell}, \mathbb{T} /\left(p^{m}, \gamma_{n}-1\right) \mathbb{T}\right) \cong H^{1}\left(k_{n}(\tau)_{\ell}, T / p^{m} T\right) .
$$

(See [C, proposition II.1.1] for (4.5) and [R3, Appendix B.5] for (4.6).) Thanks to these identifications, we may talk about the propagation of a local condition $H_{\mathcal{F}}^{1}\left(k_{\ell}, \mathbb{T}\right) \subset H^{1}\left(k_{\ell}, \mathbb{T}\right)$ at $\ell$ to a local condition

$$
H_{\mathcal{F}}^{1}\left(\left(k_{n}\right)_{\ell}, T / p^{m} T\right) \subset H^{1}\left(\left(k_{n}\right)_{\ell}, T / p^{m} T\right) \cong H^{1}\left(k_{\ell}, \mathbb{T} /\left(p^{m}, \gamma_{n}-1\right) \mathbb{T}\right) .
$$

Namely, we define $H_{\mathcal{F}}^{1}\left(\left(k_{n}\right)_{\ell}, T / p^{m} T\right)$ as the isomorphic copy of the module $H_{\mathcal{F}}^{1}\left(k_{\ell}, \mathbb{T} /\left(p^{m}, \gamma_{n}-1\right) \mathbb{T}\right)$ under isomorphism (4.6) of Shapiro's lemma.

THEOREM 4.15. We have the following.

(i) $\boldsymbol{\kappa}^{\mathrm{St}} \in \overline{\mathbf{K S}}\left(T, \mathcal{F}_{\mathcal{L}}, \mathcal{P}\right)$.

(ii) $\kappa^{\mathrm{St}} \in \overline{\mathbf{K S}}\left(\mathbb{T}, \mathcal{F}_{\mathbb{L}}, \mathcal{P}\right)$.

Proof. These are identical to the proofs of [B1, Theorem 2.19] and [B2, Theorem 3.23]. We remark that the only essential point beyond [R3] and [MR1] is to verify that

$$
\operatorname{loc}_{p}\left(\kappa_{\tau, m}^{\mathrm{St}}\right) \in H_{\mathcal{F}_{\mathcal{L}}}^{1}\left(k_{p}, T / p^{m} T\right) \cong \mathcal{L} / p^{m} \mathcal{L}
$$

for each $\tau \in \mathcal{N}_{m}$ and $m \in \mathbb{Z}^{+}$, and that

$$
\begin{aligned}
\operatorname{loc}_{p}\left(\kappa_{\tau}^{\mathrm{St}_{\infty}}(m, n)\right) & \in H_{\mathcal{F}_{\mathbb{L}}}^{1}\left(k_{p}, \mathbb{T} /\left(p^{m}, \gamma_{n}-1\right) \mathbb{T}\right) \\
& \cong \mathbb{L} /\left(p^{m}, \gamma_{n}-1\right) \mathbb{L}:=\mathcal{L}_{n} / p^{m} \mathcal{L}_{n}
\end{aligned}
$$

for every $\tau \in \mathcal{N}_{m+n}$ and every $m, n \in \mathbb{Z}^{+}$. As in [B1] and [B2], the key point in proving assertions (4.7) and (4.8) is the fact that $\mathbf{c}^{\mathrm{St}}$ is an $\mathcal{L}$-restricted Euler system. 
We give the main applications of our construction in Section 5. This will be twofold. The first application is somewhat standard; we will bound the dual Selmer groups. As the second application, we will relate the Stickelberger elements, making use of the first application, to the conjectural Rubin-Stark elements. Among other things, this will enable us to control the local behavior of Rubin-Stark elements.

REMARK 4.16. As remarked earlier, one needs only a weak Kolyvagin system in order to deduce the main applications of the $\mathcal{L}$-restricted Euler system $\mathbf{c}^{\mathrm{St}}$. See [MR1, Definition 3.1.8] for a definition of a weak Kolyvagin system. We remark that Kolyvagin's descent (see [R3, Section 4]) applied on an Euler system gives rise to a weak Kolyvagin system. A weak Kolyvagin system can be used following the formalism of [R3, Sections 5, 7] with slight alterations, to obtain the same results which we present below.

\section{$\S 5$. Applications}

Before we state our main applications of the $\mathcal{L}$-restricted Euler system $\mathbf{c}^{\mathrm{St}}$, we recall our running hypotheses. We fix a totally odd character $\chi$ of $G_{k}:=\operatorname{Gal}(\bar{k} / k)$ which is not the Teichmüller character $\omega$ (giving the action of $G_{k}$ on the $p$ th roots of unity $\boldsymbol{\mu}_{p}$ ). Throughout Section 5, we assume that (A1) holds. Suppose also that Assumption 1.1 is true.

\subsection{Main theorem over $k$}

We first prove a bound on the size of the dual Selmer group $H_{\mathcal{F}_{\mathcal{L}}^{*}}^{1}\left(k, T^{*}\right)$. We use this bound to obtain bounds on the classical (dual) Selmer groups, via the comparison theorem established in Section 2.4.1.

THEOREM 5.1. Under our running hypotheses,

(i) length $\mathbb{Z}_{p}\left(H_{\mathcal{F}_{\mathcal{L}}^{*}}^{1}\left(k, T^{*}\right)\right) \leq \operatorname{length}_{\mathbb{Z}_{p}}\left(H_{\mathcal{F}_{\mathcal{L}}}^{1}(k, T) / \mathbb{Z}_{p} \cdot \kappa_{1}^{\mathrm{St}}\right)$,

(ii) length $_{\mathbb{Z}_{p}}\left(H_{\mathcal{F}_{\mathrm{cl}}^{*}}^{1}\left(k, T^{*}\right)\right) \leq \operatorname{length}_{\mathbb{Z}_{p}}\left(\mathcal{L} / \mathbb{Z}_{p} \cdot c_{k}^{\mathrm{St}}\right)$.

Proof. (i) is Theorem 2.31. (ii) follows from (i) and Corollary 2.22 applied with $c=c_{k}^{\mathrm{St}}=\kappa_{1}^{\mathrm{St}}$.

Let $\theta_{L}^{\chi} \in \mathbb{Z}_{p}[\Delta]^{\chi}$ be as in Section 3. The evaluation map

$$
\chi: \mathbb{Z}_{p}[\Delta]^{\chi} \longrightarrow \mathbb{Z}_{p}
$$

induces an isomorphism, and we write $\chi\left(\theta_{L}\right)$ for the image of $\theta_{L}^{\chi}$ under this map. Recall the definition of $\varphi$ and $\lambda$, which we used in Sections 3 and 4 to define $\mathbf{c}_{k}^{\text {St }}$. Recall also that $\lambda=\varphi$ by definition. 
THEOREM 5.2. Under the assumptions above,

$$
\left|A_{L}^{\chi}\right| \leq\left|\mathbb{Z}_{p} / \chi\left(\theta_{L}\right) \mathbb{Z}_{p}\right|
$$

Proof. By Proposition $2.13, H_{\mathcal{F}_{c l}^{*}}^{1}\left(k, T^{*}\right) \cong A_{L}^{\chi}$, and by construction, $c_{k}^{\mathrm{St}}=$

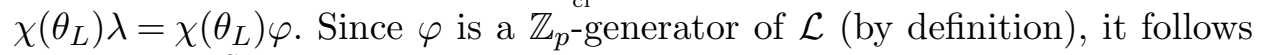
that $\mathcal{L} / \mathbb{Z}_{p} \cdot c_{k}^{\mathrm{St}}=\mathbb{Z}_{p} / \chi\left(\theta_{L}\right) \mathbb{Z}_{p}$. The proof now follows from Theorem 5.1(ii).

The inequality of Theorem 5.2 may be strengthened to an equality.

TheOREm 5.3. As in Theorem 5.2 above, assume that (A1) holds and that Assumption 1.1 is true. Suppose in addition either

(i) that $\boldsymbol{\mu}_{p} \not \subset L$, or

(ii) that the statement of Theorem 5.2 is true for $\chi=\omega$.

Then

$$
\left|A_{L}^{\chi}\right|=\left|\mathbb{Z}_{p} / \chi\left(\theta_{L}\right) \mathbb{Z}_{p}\right|
$$

Proof. Unless $\boldsymbol{\mu}_{p} \subset L$ (i.e., if we are in case (i) above), the claimed equality follows from the inequality of Theorem 5.2 using a standard argument involving the class number formula (see [R1, Section 5] and [B1, Section 3] for details). Note that we need the assumption that $\boldsymbol{\mu}_{p} \not \subset L$ for this portion since otherwise we would need the inequality of Theorem 5.2 also for the Teichmüller character $\omega$, and this escapes the methods of the current paper.

When $\boldsymbol{\mu}_{p} \subset L$, Theorem 5.2 used along with assumption (ii) gives again the desired equality utilizing the class number formula.

We note that Wiles's result [W1, Theorem 3] (only for the case $\chi=\omega$ ) is exactly the statement of Theorem 5.2 in the case $\chi=\omega$. Therefore, condition (ii) in the statement of Theorem 5.3 is true if we assume Wiles's result in this particular case.

We henceforth assume [W1, Theorem 3] (only for the case $\chi=\omega$ ) if $\boldsymbol{\mu}_{p} \subset L$.

REMARK 5.4. Note that all the Euler factors at primes $\wp \mid p$ are excluded in the definition of $\theta_{K}^{\chi}$, for any $K \in \mathcal{K}$ (recall the collection $\mathcal{K}$ from Section 3 ), contrary to the standard definition of Stickelberger elements when $K / k$ is unramified at a certain prime above $p$. Theorem 5.3 is still equivalent to [W1, Theorem 3], since assumption (A1) assures that our Stickelberger elements agree with that of $[\mathrm{W} 1]$ and $[\mathrm{Ku}]$ up to units. 


\subsection{Main theorem over $k_{\infty}$}

Along with the assumptions above, suppose also that (A2) holds. Write $\operatorname{char}(M)$ for the characteristic ideal of a torsion $\Lambda$-module $M$.

We proceed as in the previous section. First, we prove a bound for the characteristic ideal of the dual Selmer group $H_{\mathcal{F}_{\mathbb{L}}^{*}}^{1}\left(k, \mathbb{T}^{*}\right)^{\vee}$. We then use this bound, together with Proposition 2.23, to obtain a bound on the characteristic ideal of (the Pontryagin dual of) the classical (dual) Selmer groups.

THEOREM 5.5. Under the running assumptions,

$$
\operatorname{char}\left(H_{\mathcal{F}_{\mathbb{L}}^{*}}^{1}\left(k, \mathbb{T}^{*}\right)^{\vee}\right) \mid \operatorname{char}\left(H_{\mathcal{F}_{\mathbb{L}}}^{1}(k, \mathbb{T}) / \Lambda \cdot \kappa_{1}^{\mathrm{St} \infty}\right) .
$$

Proof. This is Theorem 2.38.

Set $c_{k_{\infty}}^{\mathrm{St}}:=\left\{c_{k_{n}}^{\mathrm{St}}\right\}_{n} \in \lim _{n} H^{1}\left(k_{n}, T\right)=H^{1}(k, \mathbb{T})$.

COROLlary 5.6. $\operatorname{char}\left(\left(\lim _{\longrightarrow} A_{L_{n}}^{\chi}\right)^{\vee}\right) \mid \operatorname{char}\left(\mathbb{L} / \Lambda \cdot c_{k_{\infty}}^{\mathrm{St}}\right)$.

Proof. This follows from Theorem 5.5 and Proposition 2.23(ii) applied with $c=c_{k_{\infty}}^{\mathrm{St}}$, together with the fact that $\kappa_{1}^{\mathrm{St}}=c_{k_{\infty}}^{\mathrm{St}_{\infty}}$.

Recall the element $\theta_{L_{n}}^{\chi} \in \mathbb{Z}_{p}\left[\Delta \times \Gamma_{n}\right]^{\chi}=\mathbb{Z}_{p}[\Delta]^{\chi}\left[\Gamma_{n}\right]$ from Section 3. We denote the image of $\theta_{L_{n}}^{\chi}$ under the map

$$
\chi_{\Lambda}: \mathbb{Z}_{p}[\Delta]^{\chi}\left[\Gamma_{n}\right] \longrightarrow \mathbb{Z}_{p}\left[\Gamma_{n}\right]
$$

(which extends $\chi: \mathbb{Z}_{p}[\Delta]^{\chi} \rightarrow \mathbb{Z}_{p}$ from the previous section to $\Gamma_{n}$ by letting $\chi_{\Lambda}(\gamma)=\gamma$ for $\left.\gamma \in \Gamma_{n}\right)$ by $\chi_{\Lambda}\left(\theta_{L_{n}}\right)$. Lemma 3.1 shows that $\left\{\chi_{\Lambda}\left(\theta_{L_{n}}\right)\right\}$ is a projective system with respect to the natural surjections $\mathbb{Z}_{p}\left[\Gamma_{n^{\prime}}\right] \rightarrow \mathbb{Z}_{p}\left[\Gamma_{n}\right]$, $n^{\prime} \geq n$. We define

$$
\chi_{\Lambda}\left(\Theta_{L_{\infty}}\right):=\left\{\chi_{\Lambda}\left(\theta_{L_{n}}\right)\right\} \in \underset{n}{\lim } \mathbb{Z}_{p}\left[\Gamma_{n}\right]=\Lambda
$$

Finally, let $x \mapsto x^{\bullet}$ be the involution on $\Lambda$ induced from $\gamma \mapsto \gamma^{-1}$ for $\gamma \in \Gamma$.

THEOREM 5.7. Under the running hypotheses of this section,

$$
\operatorname{char}\left(\left(\underset{n}{\lim } A_{L_{n}}^{\chi}\right)^{\vee}\right) \mid \chi_{\Lambda}\left(\Theta_{L_{\infty}}\right)^{\bullet}
$$

Proof. By the construction of $c_{k_{n}}^{\mathrm{St}}$ and $\lambda_{n}$,

$$
c_{k_{n}}^{\mathrm{St}}=\lambda_{n} \circ \theta_{L_{n}}^{\chi}=\chi_{\Lambda}\left(\theta_{L_{n}}\right)^{\bullet} \lambda_{n}=\chi_{\Lambda}\left(\theta_{L_{n}}\right)^{\bullet} \varphi_{n} .
$$


It follows that

$$
c_{k_{\infty}}^{\mathrm{St}}=\left\{\chi_{\Lambda}\left(\theta_{L_{n}}\right)^{\bullet} \varphi_{n}\right\}_{n}=\chi_{\Lambda}\left(\Theta_{L_{\infty}}\right)^{\bullet} \Phi
$$

with $\Phi=\left\{\varphi_{n}\right\}$ as in Section 4.1. Since $\Phi$ is a generator of $\mathbb{L}$ (by definition), the theorem follows from Corollary 5.6.

Once again, making use of a standard class number argument (and yet again, the case $\boldsymbol{\mu}_{p} \subset L$ requires more care as above) shows that this equality may be turned into an equality, as follows.

TheOREM 5.8. Under the hypotheses of Theorem 5.3 and assuming (A2),

$$
\operatorname{char}\left(\left(\underset{n}{\lim } A_{L_{n}}^{\chi}\right)^{\vee}\right)=\chi_{\Lambda}\left(\Theta_{L_{\infty}}\right)^{\bullet} .
$$

Let $\rho_{\text {cyc }}: G_{k} \rightarrow \mathbb{Z}_{p}^{\times}$be the cyclotomic character (giving the action of $G_{k}$ on $\left.\boldsymbol{\mu}_{p^{\infty}}\right)$, and set $\left\langle\rho_{\text {cyc }}\right\rangle=\omega^{-1} \rho_{\text {cyc }}: \Gamma \rightarrow 1+p \mathbb{Z}_{p}$. We define a twisting map $\mathrm{Tw}_{\left\langle\rho_{\text {cyc }}\right\rangle}: \Lambda \rightarrow \Lambda$ by setting

$$
\operatorname{Tw}_{\left\langle\rho_{\text {cyc }}\right\rangle}(\gamma)=\left\langle\rho_{\text {cyc }}\right\rangle(\gamma) \gamma \text { for } \gamma \in \Gamma
$$

and extending to $\Lambda$ by linearity and continuity. Finally, let $\mathcal{L}_{\omega \chi^{-1}} \in \Lambda$ denote the Deligne-Ribet $p$-adic L-function for the character $\omega \chi^{-1}$. We will loosely say here that $\mathcal{L}_{\omega \chi^{-1}}$ is characterized by the interpolation property

$$
\left\langle\rho_{\text {cyc }}\right\rangle^{k} \xi\left(\mathcal{L}_{\omega \chi^{-1}}\right)=\prod_{\wp \mid p}\left(1-\omega^{-k} \xi \chi(\wp) \mathbf{N}_{\wp}{ }^{k-1}\right) L\left(1-k, \omega^{-k} \xi \chi\right)
$$

for every $k \geq 1$ and every character $\xi$ of $\Gamma$ of finite order. Here, $L(s, \varrho)$ is the (abelian) Artin $L$-function attached to a character $\varrho$ of $G_{k}$ which is of finite order.

Lemma 5.9. $\chi_{\Lambda}\left(\Theta_{L_{\infty}}\right)^{\bullet}=\mathrm{Tw}_{\left\langle\rho_{\text {cyc }}\right\rangle}\left(\mathcal{L}_{\omega \chi^{-1}}\right)$.

Proof. For every character $\xi$ of $\Gamma$ of finite order, it follows from the definitions that

$$
\begin{aligned}
\xi\left(\chi_{\Lambda}\left(\Theta_{L_{\infty}}\right)^{\bullet}\right)=\xi^{-1}\left(\chi_{\Lambda}\left(\Theta_{L_{\infty}}\right)\right) & =\prod_{\wp \mid p}\left(1-\chi^{-1} \xi(\wp)\right) L\left(0, \chi^{-1} \xi\right) \\
& =\left\langle\rho_{\text {cyc }}\right\rangle \xi\left(\mathcal{L}_{\omega \chi^{-1}}\right)=\xi\left(\left\langle\rho_{\text {cyc }}\right\rangle \mathcal{L}_{\omega \chi^{-1}}\right) .
\end{aligned}
$$

Since this is true for every $\xi$, the lemma follows.

Corollary 5.10. Under the assumptions of Theorem 5.8,

$$
\operatorname{char}\left(\left(\underset{n}{\lim } A_{L_{n}}^{\chi}\right)^{\vee}\right)=\operatorname{Tw}_{\left\langle\rho_{\text {cyc }}\right\rangle}\left(\mathcal{L}_{\omega \chi^{-1}}\right)
$$




\subsection{Twisting and local Iwasawa theory of Stark elements}

The goal of this section is to establish a connection between the Stickelberger elements and the conjectural Rubin-Stark elements. To achieve this, we will employ the following two ingredients.

(1) The twisting formalism developed in [R3, Section VI].

(2) The rigidity statement in [B2, Theorem 2.19(ii)] for the module of $\Lambda$ adic Kolyvagin systems.

Write $\rho:=\left\langle\rho_{\text {cyc }}\right\rangle$ for notational simplicity. Let $\psi=\omega \chi^{-1}$ as in Section 1, and set

$$
T^{\prime}=\mathbb{Z}_{p}(1) \otimes \psi^{-1}=T \otimes \rho .
$$

We also write $\mathbb{T}^{\prime}=T^{\prime} \otimes \Lambda$.

\subsubsection{Twisting argument.}

LEMMA 5.11. Suppose that $\rho$ is as above.

(i) There is a commutative diagram

$$
\begin{aligned}
& \lim _{n} H^{1}\left(k_{n}, T\right) \otimes \rho \longrightarrow \lim _{n} H^{1}\left(k_{n}, T^{\prime}\right) \\
& \operatorname{loc}_{p} \downarrow \quad \downarrow \operatorname{loc}_{p} \\
& \lim _{n} H^{1}\left(\left(k_{n}\right)_{p}, T\right) \otimes \rho \stackrel{\sim}{\longrightarrow} \underset{n}{\lim } H^{1}\left(\left(k_{n}\right)_{p}, T^{\prime}\right)
\end{aligned}
$$

such that the horizontal arrows are natural isomorphisms.

(ii) There is a commutative diagram

$$
\begin{aligned}
& \lim _{\overleftarrow{K \in \mathcal{K}_{0}}} H^{1}(K, T) \otimes \rho \stackrel{\sim}{\longrightarrow} \lim _{K \in \mathcal{K}_{0}} H^{1}\left(K, T^{\prime}\right) \\
& \operatorname{loc}_{p} \downarrow \quad \downarrow \operatorname{loc}_{p} \\
& \lim _{K \in \mathcal{K}_{0}} H^{1}\left(K_{p}, T\right) \otimes \rho \stackrel{\sim}{\longrightarrow} \lim _{K \in \mathcal{K}_{0}} H^{1}\left(K_{p}, T^{\prime}\right)
\end{aligned}
$$

such that the horizontal arrows are natural isomorphisms.

(iii) $\left(\lim _{\longrightarrow} H_{\mathcal{F}_{\mathrm{cl}}^{*}}^{1}\left(k_{n}, T^{*}\right)\right)^{\vee} \otimes \rho \stackrel{\sim}{\longrightarrow}\left(\lim _{\longrightarrow} H_{\mathcal{F}_{\mathrm{cl}}^{*}}^{1}\left(k_{n},\left(T^{\prime}\right)^{*}\right)\right)^{\vee}$.

Proof. This is [R3, Proposition VI.2.1]. We note that $\lim _{\longrightarrow} H_{\mathcal{F}_{\mathrm{cl}}^{*}}^{1}\left(k_{n}, T^{*}\right)$ here coincides with what Rubin calls $\mathcal{S}_{\Sigma_{p}}\left(k_{\infty}, W\right)$, thanks to Remark 2.12, where $W=T^{*}$ and where $\Sigma_{p}$ is the set of places of $k$ which lie above $p$. 
Let $\mathcal{L} \subset \lim _{K \in \mathcal{K}_{0}} H^{1}\left(K_{p}, T\right)$ be as in Section 4.7, and let $\mathcal{L}_{\rho}$ denote the image of $\mathcal{L}$ under the isomorphism of Lemma 5.11(ii). Recall the $\mathcal{L}$-restricted Euler system $\mathbf{c}^{\mathrm{St}}$ that we constructed at the end of Section 4.1. Let $\mathbf{c}^{\mathrm{St}, \rho}$ denote the twist of the Euler system $\mathbf{c}^{\mathrm{St}}$ defined via [R3, Theorem VI.3.5]; this means that $\mathbf{c}^{\mathrm{St}, \rho} \in \mathbf{E S}\left(T^{\prime}, \mathcal{K}\right)$. Furthermore, one can see without difficulty that the following lemma is true.

LEMMA 5.12. The twisted Euler system $\mathbf{c}^{\mathrm{St}, \rho}$ is $\mathcal{L}_{\rho}$-restricted.

Let $\mathbb{L}_{\rho} \in H^{1}\left(k_{p}, \mathbb{T}^{\prime}\right)$ denote the image of $\mathcal{L}_{\rho}$ under the obvious projection map. Recall the element $c_{k_{\infty}}^{\mathrm{St}}=\left\{c_{k_{n}}^{\mathrm{St}}\right\}_{n} \in H^{1}(k, \mathbb{T})$, and set $c_{k_{\infty}}^{\mathrm{St}, \rho}=\left\{c_{k_{n}}^{\mathrm{St}, \rho}\right\}_{n} \in$ $H^{1}\left(k, \mathbb{T}^{\prime}\right)$. Note that $\operatorname{loc}_{p}\left(c_{k_{\infty}}^{\mathrm{St}}\right) \in \mathbb{L} \subset H^{1}\left(k_{p}, \mathbb{T}\right)$ and $\operatorname{loc}_{p}\left(c_{k_{\infty}}^{\mathrm{St}, \rho}\right) \in \mathbb{L}_{\rho} \subset$ $H^{1}\left(k_{p}, \mathbb{T}^{\prime}\right)$, by construction. As before, we drop $\operatorname{loc}_{p}$ from notation and denote $\operatorname{loc}_{p}\left(c_{k_{\infty}}^{\mathrm{St}}\right)\left(\operatorname{resp} ., \operatorname{loc}_{p}\left(c_{k_{\infty}}^{\mathrm{St}, \rho}\right)\right)$ simply by $c_{k_{\infty}}^{\mathrm{St}}$ (resp., by $\left.c_{k_{\infty}}^{\mathrm{St}, \rho}\right)$. Lemma 5.11(i) induces an isomorphism,

$$
\mathbb{L} / \Lambda \cdot c_{k_{\infty}}^{\mathrm{St}} \otimes \rho \stackrel{\sim}{\longrightarrow} \mathbb{L}_{\rho} / \Lambda \cdot c_{k_{\infty}}^{\mathrm{St}, \rho}
$$

To simplify notation, set $X_{\infty}(T):=\left(\varliminf_{\lim _{n}} H_{\mathcal{F}_{\mathrm{cl}}^{*}}^{1}\left(k_{n}, T^{*}\right)\right)^{\vee}$, and set similarly $X_{\infty}\left(T^{\prime}\right):=\left(\lim _{\longrightarrow} H_{\mathcal{F}_{\mathrm{cl}}^{*}}^{1}\left(k_{n},\left(T^{\prime}\right)^{*}\right)\right)^{\vee}$. Until the end of this paper, assume that (A1) and (A2) both hold true.

Proposition 5.13. Let $\operatorname{Tw}_{\rho}: \Lambda \rightarrow \Lambda$ be the twisting operator as above.

(i) $\operatorname{Tw}_{\rho}\left(\operatorname{char}\left(X_{\infty}\left(T^{\prime}\right)\right)\right)=\operatorname{char}\left(X_{\infty}(T)\right)$.

(ii) $\operatorname{Tw}_{\rho}\left(\operatorname{char}\left(\mathbb{L}_{\rho} / \Lambda \cdot c_{k_{\infty}}^{\mathrm{St}, \rho}\right)\right)=\operatorname{char}\left(\mathbb{L} / \Lambda \cdot c_{k_{\infty}}^{\mathrm{St}}\right)$.

(iii) $\operatorname{char}\left(X_{\infty}\left(T^{\prime}\right)\right)=\operatorname{char}\left(\mathbb{L}_{\rho} / \Lambda \cdot c_{k_{\infty}}^{\mathrm{St}, \rho}\right)$.

Proof. Assertion (i) follows from Lemma 5.11(iii) together with [R3, Lemma VI.1.2(i)], and (ii) from (5.3) applied with [R3, Lemma VI.1.2(i)].

Thanks to (i) and (ii), assertion (iii) is equivalent to verifying that

$$
\operatorname{Tw}_{\rho^{-1}}\left(\operatorname{char}\left(X_{\infty}(T)\right)\right)=\operatorname{Tw}_{\rho^{-1}}\left(\operatorname{char}\left(\mathbb{L} / \Lambda \cdot c_{k_{\infty}}^{\mathrm{St}}\right)\right) .
$$

This, however, is the statement of Theorem 5.8 put together with (5.1) and twisted by $\mathrm{Tw}_{\rho^{-1}}$.

5.3.2. Stickelberger elements versus Rubin-Stark elements. Following [B2, Definition 2.10], we define the $\mathbb{L}_{\rho}$-modified Selmer structure $\mathcal{F}_{\mathbb{L}_{\rho}}$ on $\mathbb{T}^{\prime}$, for $\mathbb{L}_{\rho} \subset H^{1}\left(k_{p}, \mathbb{T}^{\prime}\right)$ as above. The argument of [B2, Theorem 3.23] shows that the $\mathcal{L}_{\rho}$-restricted Euler system $\mathbf{c}^{\mathrm{St}, \rho}$ gives rise to a Kolyvagin system $\boldsymbol{\kappa}^{\mathrm{St}}, \rho \in \overline{\mathbf{K S}}\left(\mathbb{T}^{\prime}, \mathcal{F}_{\mathbb{L}_{\rho}}, \mathcal{P}\right)$. 
Proposition 5.14. We have

$$
\operatorname{char}\left(H_{\mathcal{F}_{\mathbb{L}_{\rho}^{*}}^{*}}^{1}\left(k,\left(\mathbb{T}^{\prime}\right)^{*}\right)^{\vee}\right)=\operatorname{char}\left(H_{\mathcal{F}_{\mathbb{L}_{\rho}}}^{1}\left(k, \mathbb{T}^{\prime}\right) / \Lambda \cdot \kappa_{1}^{\mathrm{St}}{ }^{\mathrm{St}, \rho}\right)
$$

Proof. This follows from Proposition 5.13 and the exact sequence of Proposition 2.23(ii) rewritten for $\mathbb{T}^{\prime}$ instead of $\mathbb{T}$. See also [B2, Proposition 2.12] for this version of the exact sequence of Proposition 2.23(ii).

Assume until the end of this paper that the character $\psi$ satisfies hypothesis (A1) as well, that is, that $\psi(\wp) \neq 1$ for any prime $\wp$ of $k$ lying above $p$. According to [B2, Theorem 2.19(ii)], the $\Lambda$-module of Kolyvagin systems $\overline{\mathbf{K S}}\left(\mathbb{T}^{\prime}, \mathcal{F}_{\mathbb{L}_{\rho}}, \mathcal{P}\right)$ is free of rank 1 .

COROLlary 5.15. The Kolyvagin system $\boldsymbol{\kappa}^{\mathrm{St}_{\infty}, \rho}$ generates the cyclic module $\overline{\mathbf{K S}}\left(\mathbb{T}^{\prime}, \mathcal{F}_{\mathbb{L}_{\rho}}, \mathcal{P}\right)$.

Proof. We know by [B2, Theorem 2.19(ii), Proposition 4.2] that the free $\Lambda$-module $\overline{\mathbf{K S}}\left(\mathbb{T}^{\prime}, \mathcal{F}_{\mathbb{L}_{\rho}}, \mathcal{P}\right)$ is generated by a $\Lambda$-primitive Kolyvagin system $\boldsymbol{\kappa}$ (in the sense of [MR1, Definition 5.3.9]). In particular, we may write

$$
\boldsymbol{\kappa}^{\mathrm{St}_{\infty}, \rho}=u \cdot \boldsymbol{\kappa}
$$

with $u \in \Lambda$.

The main application of the $\Lambda$-primitive Kolyvagin system $\kappa$ for the Selmer triple $\left(\mathbb{T}^{\prime}, \mathcal{F}_{\mathbb{L}_{\rho}}, \mathcal{P}\right)$ is the following (see [B2, Theorem 2.20]):

$$
\operatorname{char}\left(H_{\mathcal{F}_{\mathbb{L}_{\rho}^{*}}}^{1}\left(k,\left(\mathbb{T}^{\prime}\right)^{*}\right)^{\vee}\right)=\operatorname{char}\left(H_{\mathcal{F}_{\mathbb{L}_{\rho}}}^{1}\left(k, \mathbb{T}^{\prime}\right) / \Lambda \cdot \kappa_{1}\right)
$$

Assertion (5.5) together with Proposition 5.14 shows that $u \in \Lambda^{\times}$. This completes the proof.

Let $\mathfrak{e}^{\text {Stark }}=\left\{\mathfrak{e}^{\text {Stark }}\right\}_{K \in \mathcal{K}_{0}}$ be the Euler system $\left\{\varepsilon_{K, \Phi_{0}^{(\infty)}}^{\psi}\right\}_{K \in \mathcal{K}_{0}}$ defined in [B2, Proposition 3.14]. This Euler system is obtained from the Rubin-Stark elements that Rubin [R2] conjectured to exist, and therefore the existence of $\boldsymbol{e}^{\text {Stark }}$ is implicitly assumed here.* Note that our $\psi$ here is denoted by $\chi$ in $[\mathrm{B} 2]$. Let

$$
c_{k_{\infty}}^{\mathrm{Stark}} \in \wedge^{r} H^{1}\left(k_{p}, \mathbb{T}^{\prime}\right)
$$

${ }^{*}$ Having said that, note that we will recover in Theorem 5.16(i) below the Kolyvagin system $\boldsymbol{\kappa}^{\text {Stark }}$ up to a unit (which descends from $\boldsymbol{e}^{\text {Stark }}$ ) directly from the Kolyvagin system $\kappa^{\mathrm{St}} \infty$, which is obtained in this article from Stickelberger elements. 
be the element defined as in [B2, Remark 4.5]. Finally, let $\boldsymbol{\kappa}^{\text {Stark }}$ be the $\Lambda$-primitive Kolyvagin system for the Selmer triple $\left(\mathbb{T}^{\prime}, \mathcal{F}_{\mathbb{L}_{\rho}}, \mathcal{P}\right)$, which is obtained from the Euler system $\mathfrak{e}^{\text {Stark }}$. We remark that what we call $\mathbb{L}_{\rho}$ here is denoted by $\mathbb{L}_{\infty}$ in [B2], and $\boldsymbol{\kappa}^{\text {Stark }}$ here is denoted by $\boldsymbol{\kappa}^{\Phi_{0}^{(\infty)}}$ in [B2]. Recall the Kolyvagin system $\boldsymbol{\kappa}^{\mathrm{St}{ }_{\infty}, \rho}$ which is obtained from the $\mathcal{L}_{\rho}$-restricted Euler system $\mathbf{c}^{\mathrm{St}, \rho}$ of Stickelberger elements.

The theorem below draws a connection between the Stickelberger elements and the Rubin-Stark elements.

THEOREM 5.16. The following hold under the running assumptions:

(i) there is a unit $u \in \Lambda^{\times}$such that $\boldsymbol{\kappa}^{\mathrm{St}_{\infty}, \rho}=u \cdot \boldsymbol{\kappa}^{\mathrm{Stark}}$,

(ii) $\operatorname{char}\left(\mathbb{L}_{\rho} / \Lambda \cdot \kappa_{1}^{\mathrm{St}_{\infty}, \rho}\right)=\operatorname{char}\left(\mathbb{L}_{\rho} / \kappa_{1}^{\mathrm{Stark}}\right)$,

(iii) $\operatorname{char}\left(\wedge^{r} H^{1}\left(k_{p}, \mathbb{T}^{\prime}\right) / \Lambda \cdot c_{k_{\infty}}^{\text {Stark }}\right)=\mathcal{L}_{\psi}$.

Here, $\mathcal{L}_{\psi}$ is the Deligne-Ribet p-adic L-function attached to the totally even character $\psi$.

Proof. Assertion (i) follows from Corollary 5.15 and the fact that $\boldsymbol{\kappa}^{\text {Stark }}$ generates the free rank $1 \Lambda$-module $\overline{\mathbf{K S}}\left(\mathbb{T}^{\prime}, \mathcal{F}_{\mathbb{L}_{\rho}}, \mathcal{P}\right)$. Assertion (ii) is immediate from (i).

The discussion preceding the statement of [B2, Corollary 4.6] shows that

$$
\wedge^{r} H^{1}\left(k_{p}, \mathbb{T}^{\prime}\right) / \Lambda \cdot c_{k_{\infty}}^{\text {Stark }} \cong \mathbb{L}_{\rho} / \Lambda \cdot \kappa_{1}^{\text {Stark }}
$$

Hence, it follows from (ii) that

$$
\operatorname{char}\left(\wedge^{r} H^{1}\left(k_{p}, \mathbb{T}^{\prime}\right) / \Lambda \cdot c_{k_{\infty}}^{\mathrm{Stark}}\right)=\operatorname{char}\left(\mathbb{L}_{\rho} / \Lambda \cdot \kappa_{1}^{\mathrm{St}}, \rho\right) .
$$

By the construction of the Kolyvagin system $\boldsymbol{\kappa}^{\mathrm{St}, \rho}$ out of the Euler system $\mathbf{c}^{\mathrm{St}, \rho}$, it follows that $\kappa_{1}^{\mathrm{St}, \rho}=c_{k_{\infty}}^{\mathrm{St}, \rho}$, which in turn implies that

$$
\operatorname{char}\left(\wedge^{r} H^{1}\left(k_{p}, \mathbb{T}^{\prime}\right) / \Lambda \cdot c_{k_{\infty}}^{\text {Stark }}\right)=\operatorname{char}\left(\mathbb{L}_{\rho} / \Lambda \cdot c_{k_{\infty}}^{\mathrm{St}, \rho}\right) .
$$

Using Proposition 5.13(ii), (5.1), and Lemma 5.9, we see that

$$
\operatorname{Tw}_{\rho}\left(\operatorname{char}\left(\mathbb{L}_{\rho} / \Lambda \cdot c_{k_{\infty}}^{\mathrm{St}, \rho}\right)\right)=\operatorname{char}\left(\mathbb{L} / \Lambda \cdot c_{k_{\infty}}^{\mathrm{St}}\right)=\operatorname{Tw}_{\rho}\left(\mathcal{L}_{\psi}\right) .
$$

This completes the proof of the theorem.

The author [B2, Theorem 4.7] has previously deduced Theorem 5.16(iii) from Iwasawa's main conjecture. Here, we need to assume slightly less, namely, the $\chi$-part of Brumer's conjecture (see Assumption 1.1) to prove this statement. 
Acknowledgments. The author worked as a William Hodge postdoctoral fellow at the Institut des Hautes Études Scientifiques when this paper was written up, and he wishes to extend his hearty thanks for warm hospitality there. He also thanks Masato Kurihara for his interest in this work and for an encouraging discussion on the results of this article when the author visited Keio. Thanks go also to the referee for numerous comments and suggestions that improved the exposition.

\section{REFERENCES}

[B1] K. Büyükboduk, Kolyvagin systems of Stark units, J. Reine Angew. Math. 631 (2009), 85-107.

[B2] , Stark units and the main conjectures for totally real fields, Compos. Math. 145 (2009), 1163-1195.

[B3] $\longrightarrow \Lambda$-adic Kolyvagin systems, Int. Math. Res. Notices (2010), doi: 10:1093/ $\mathrm{imrn} / \mathrm{rnq} 186$.

[B4] - On Euler systems of rank $r$ and their Kolyvagin systems, Indiana Univ. Math. J. 59 (2010), 1245-1276.

[C] P. Colmez, Théorie d'Iwasawa des représentations de de Rham d'un corps local, Ann. of Math. (2) 148 (1998), 485-571.

[DR] P. Deligne and K. A. Ribet, Values of abelian L-functions at negative integers over totally real fields, Invent. Math. 59 (1980), 227-286.

[dS] E. de Shalit, Iwasawa Theory of Elliptic Curves with Complex Multiplication, Perspect. Math. 3, Academic Press, Boston, 1987.

[G] R. Greenberg, "Trivial zeros of $p$-adic L-functions" in $p$-Adic Monodromy and the Birch and Swinnerton-Dyer Conjecture (Boston, MA, 1991), Contemp. Math. 165, Amer. Math. Soc., Providence, 1994, 149-174.

[Gr] C. Greither, Computing fitting ideals of Iwasawa modules, Math. Z. 246 (2004), 733-767.

[Ka] K. Kato, Euler systems, Iwasawa theory, and Selmer groups, Kodai Math. J. 22 (1999), 313-372.

[Ku] M. Kurihara, On the structure of ideal class groups of CM-fields, Doc. Math. DMV Extra Vol (2003), 539-563.

[MR1] B. Mazur and K. Rubin, Kolyvagin systems, Mem. Amer. Math. Soc. 168 (2004), viii +96 .

[MR2] - Refined class number formulas and Kolyvagin systems, preprint, arXiv:0909.3916v1 [math.NT]

[Mi] J. S. Milne, Arithmetic Duality Theorems, Perspect. Math. 1, Academic Press, Boston, 1986.

[P1] B. Perrin-Riou, Théorie d'Iwasawa des représentations p-adiques sur un corps local, with an appendix by J.-M. Fontaine, Invent. Math. 115 (1994), 81-161.

[P2] - Systèmes d'Euler p-adiques et théorie d'Iwasawa, Ann. Inst. Fourier (Grenoble) 48 (1998), 1231-1307.

[R1] K. Rubin, Stark units and Kolyvagin's "Euler systems," J. Reine Angew. Math. 425 (1992), 141-154.

[R2] - A Stark conjecture "over $\mathbf{Z}$ " for abelian L-functions with multiple zeros, Ann. Inst. Fourier (Grenoble) 46 (1996), 33-62. 
[R3] , Euler Systems, Ann. of Math. Stud. 147, Princeton University Press, Princeton, 2000.

[S] C. L. Siegel, Über die Fourierschen Koeffizienten von Modulformen, Nachr. Akad. Wiss. Göttingen Math.-Phys. Kl. II 1970, 15-56.

[T] J. Tate, Les conjectures de Stark sur les fonctions L d'Artin en $s=0$, Progr. Math. 47, Birkhäuser, Boston, 1984.

[W1] A. Wiles, On a conjecture of Brumer, Ann. of Math. (2) 131 (1990), 555-565.

[W2] - The Iwasawa conjecture for totally real fields, Ann. of Math. (2) 131 (1990), 493-540.

Institut des Hautes Études Scientifiques

Route de Chartres

F-91440 Bures-sur-Yvette

France

Current:

Koç University

Mathematics

34450 Sariyer

Istanbul

Turkey

kbuyukboduk@ku.edu.tr 